The Astronomical Journal, 121:2618-2637, 2001 May

(C) 2001. The American Astronomical Society. All rights reserved. Printed in U.S.A.

\title{
THE METAL-RICH GLOBULAR CLUSTER NGC 6553: OBSERVATIONS WITH WFPC2, STIS, AND NICMOS ${ }^{1}$
}

\author{
Sylvie F. Beaulieu, Gerard Gilmore, Rebecca A. W. Elson, ${ }^{2}$ and Rachel A. Johnson \\ Institute of Astronomy, Madingley Road, Cambridge, CB3 0HA, England, UK; beaulieu@ast.cam.ac.uk, gil@ast.cam.ac.uk, raj@ast.cam.ac.uk \\ Basilio SANTIAGo \\ Instituto de Física, Universidade Federal do Rio Grande do Sul, 91510-970 Porto Alegre, RS, Brazil; santiago@if.ufrgs.br
}

STEINN SigURDSSON

Department of Astronomy and Astrophysics, Pennsylvania State University, University Park, PA 16802; steinn@astro.psu.edu

AND

NiAL TANVIR

Department of Physical Science, University of Hertfordshire, College Lane, Hatfield, AL10 9AB, England, UK; nrt@star.herts.ac.uk Received 2000 June 11; accepted 2001 February 8

\begin{abstract}
We present a Hubble Space Telescope study of the metal-rich globular cluster NGC 6553 using WFPC2, NICMOS, and STIS. Our primary motivation is to calibrate the STIS broadband LP magnitude against $V_{555}$ and $I_{814}$ magnitudes for stars of known metallicity and absolute (visual) magnitude, for application to our study of LMC globular clusters. NGC 6553 has been shown in earlier studies to have a very unusual color-magnitude diagram, so we also use our data to investigate the reddening, distance, luminosity function, and structure of this cluster. We deduce a higher metallicity and smaller distance modulus than did some previous studies, but we emphasize that very large patchy extinction on small angular scales prohibits accurate determination of the parameters of this cluster. The horizontal branch of NGC 6553 in $(V, V-I)$ is tilted at an angle close to that of the reddening vector. We show that extinction does not, however, explain the tilt, which is presumably a metallicity effect. The colormagnitude diagram shows an apparent second turnoff some 1.5 mag fainter than that of the cluster. We show that this is most likely the background Galactic bulge: however, in that case, the color-magnitude diagram of NGC 6553 is not a good match to that of the field bulge population. The cluster is probably more metal-rich than is the mean field bulge star.
\end{abstract}

Key words: cosmic rays - galaxies: luminosity function, mass function - globular clusters: general globular clusters: individual (NGC 6553)

\section{INTRODUCTION}

This paper is one of a series describing the results of a large Hubble Space Telescope (HST) project to study the formation and evolution of rich star clusters in the Large Magellanic Cloud (LMC). The Galactic bulge globular cluster, NGC 6553, was observed as part of this program primarily for calibration purposes, in order to transform NICMOS and STIS magnitudes for LMC cluster stars directly into absolute magnitudes. Using Galactic globular clusters observed in all the $H S T$ instruments directly ensures a calibration known to be appropriate to the specific stellar absolute magnitude, temperature, and metallicity. This is particularly important for determining the low-mass end of the luminosity function in the LMC clusters, one of our primary goals. By choosing nearby Galactic clusters, we are able to use as templates many stars with relatively well-determined properties, with an exposure time short compared with that required in the LMC.

NGC 6553 was chosen as a suitable metal-rich template cluster on the basis of its several detailed analyses (most

\footnotetext{
${ }^{1}$ Based on observations with the NASA/ESA Hubble Space Telescope, obtained at the Space Telescope Science Institute, which is operated by the Association of Universities for Research in Astronomy, Inc., under NASA contract NAS 5-26555. No. 9 .
}

recently that of Guarnieri et al. 1998) and because of its measured metallicity of $[\mathrm{Fe} / \mathrm{H}]=-0.22 \pm 0.05$, representative of the metallicities of the young and intermediate age LMC clusters (see Olszewski et al. 1991). It later became clear, from reanalysis of archival HST data (Feltzing \& Gilmore 2000), that this cluster has some observational difficulties, particularly very large and nonuniform extinction, which make it a less than ideal calibrator. Nonetheless, it remains a relatively metal-rich globular cluster.

Beyond calibration, our data allow us to reinvestigate the observational properties of the cluster itself. Clusters like NGC 6553 are of particular interest in that, being old and metal-rich, they are the only convenient single stellar populations representing a typical star in giant galaxies. Metalrich clusters are used as a tracer of the formation and evolution of either the Galactic bulge (in spite of their relatively large distance from the bulge), the late evolution of the halo (in spite of their high metallicity and possibly inappropriate kinematics), or the early evolution of the disk (in spite of the failure of the disk to make younger globulars). More importantly, they extend the metallicity range over which one may determine cluster luminosity (and mass) functions to metallicities typical of most stars. In this regard, the properties of the metal-rich clusters provide clues concerning the enrichment history of the inner Galaxy, the timescale for its formation, and the time of its formation relative to the halo and thick disk. Their dynamically vulnerable location also allows the effect of tidal 
shocking on the structure and stellar content of the clusters to be explored.

Our WFPC2 data allow us to probe the stellar content of NGC 6553 well below its main-sequence turnoff, and they allow another investigation of the cluster's distance and reddening. Our STIS data make it possible for us to determine a deeper luminosity function than that previously available, which may be compared with luminosity functions in other globular clusters. The combination of our optical and near-IR NICMOS data allows checks on the nature of the very tilted "horizontal" branch, testing the relative importances of extinction and astrophysical explanations, following Ortolani et al. (1990).

Two WFPC2 studies of NGC 6553 have been published; a detailed study by Guarnieri et al. (1998) and an analysis as part of a larger study of the age of the bulge by Feltzing \& Gilmore (2000). The former is based on F555W and F814W exposures of 200 and $100 \mathrm{~s}$, respectively $\left(\frac{1}{3}\right.$ and $\frac{1}{6}$ the length of our exposures). The latter is based on archive data, primarily that of Guarnieri et al. (1998), supplemented by observations in other lines of sight. Feltzing \& Gilmore (2000) provide a summary of the very many determinations of the metallicity, distance, and extinction toward NGC 6553 that are available in the literature. Since the precise values of these parameters are of secondary importance here, we refer to that paper for details and references to the original work. The several ground-based photometric studies are discussed and reviewed in Sagar et al. (1999).

Results derived from our WFPC 2 data are described in $\S$ 2. Our STIS data, together with a deep luminosity function for NGC 6553, are described in $\S 3$. NICMOS data are described in $\S 4$. Section 5 contains relations between stellar magnitudes in the various filters, $V_{555}, I_{814}$, and $I_{\mathrm{LP}}$.

\subsection{The HST/LMC Globular Cluster Project}

HST project GO 7307 is a study of the evolution of rich star clusters in the LMC. A total of 95 orbits was allocated to this project in Cycle 7, using all of the three imaging instruments - the Wide Field and Planetary Camera 2 (WFPC2), the Space Telescope Imaging Spectrograph (STIS) (in imaging mode), and the near-infrared camera and multiobject spectrometer (NICMOS) - in parallel to obtain multiwavelength photometry in eight clusters with ages $\sim 10^{7}, 10^{8}, 10^{9}$, and $10^{10} \mathrm{yr}$. In addition, shorter exposure observations of one Galactic globular cluster obtained here, as well as observations of two other clusters coordinated with another HST GO project (7419, Feltzing, Gilmore, \& Wyse 1999; Houdashelt, Wyse, \& Gilmore 2000), are being used to provide an empirical calibration of the transformation from "native" STIS and NICMOS magnitudes to standard absolute magnitudes. The three clusters include one very metal poor (M15), one of intermediate metallicity (47 Tuc), and one metal-rich (NGC 6553). By providing STIS and NICMOS observations of stars of known metallicity and absolute (visual) magnitude in these calibration clusters, we are able to derive this calibration directly, comparing stars of similar mass and metallicity. We will apply these calibrations to our LMC cluster luminosity functions in future papers. First results have been presented by Beaulieu et al. (1999, 2000), Elson et al. (1999), and Johnson et al. $(1998,2001)$. The results of a pilot study of one of the clusters in the sample are described in Elson et al. (1998a, 1998b), and Burleigh et al. (1999). Other recent results from this project are presented in Santiago et al. (2001; WFPC2 luminosity functions for six clusters), in Castro et al. (2001; WFPC2 deep CMDs of LMC field stars), and in Johnson et al. (2000; age spreads in young LMC clusters).

A brief summary of the goals of the project is as follows. In all the clusters we are using color spreads among mainsequence stars to look for populations of close stellar binaries, both in the core and as a function of radius. We will thereby determine the fraction of primordial binaries from the youngest clusters, as well as how the binary fraction evolves as the clusters age. Binaries are believed to play a crucial dynamical role in the evolution of rich star clusters, particularly in defining the rate of evolution of the core in old age. Within the youngest clusters our observations investigate the massive stellar population, and look for age spreads. Spreads in color at faint magnitudes $(V \sim 25)$ are used to identify pre-main-sequence stars. We will thus address the questions of whether the high- or low-mass stars form sequentially or together when a gas cloud collapses to form an approximately coeval cluster of stars, and whether the timescale for star formation is much shorter than, or comparable to, the dynamical crossing time.

We are determining deep luminosity functions (LFs) in all the clusters both in the cores and further out. In the youngest clusters this will indicate whether there is primordial mass segregation with, for instance, the most massive stars forming preferentially in the deepest part of the protocluster's potential well. In the older clusters this will enable us to trace the development of mass segregation, as the heaviest stars sink to the center, and investigate the formation and evolution of the blue straggler population(s). Finally, since the stellar populations in the young and intermediate age clusters are relatively unaffected by dynamical selection, their LFs may be translated straightforwardly into initial mass functions (IMFs). We can therefore address directly the all-important question of whether these clusters display a "universal" IMF. The as-yet-unproved assumption that the IMF is universal underpins all attempts to interpret the integrated light of galaxies at cosmological distances.

\section{NGC 6553: WFPC2 PHOTOMETRY}

$V$ - and $I$-band data were obtained with WFPC2, with the cluster centered on WFC chip 3, rather than on the PC. The scale of WFPC2 chips 2, 3, and 4 is 0.0996 pixel $^{-1}$, while the PC (Chip 1) has a scale of $0^{\prime \prime} 0455$ pixel $^{-1}$. The gain was set to $7 e-\mathrm{DN}^{-1}$. Instrumental parameters and in-orbit characteristics of WFPC2 can be found in Biretta et al. (1996). NGC 6553 has a core radius $r_{c}=0.55$ and a halfmass radius $r_{h}=1.55$ (Harris 1996), so chip 3 contains somewhat more than the cluster core, while the full WFPC2 field of view extends to slightly more than $r_{h}$. Table 1 summaries the basic properties of NGC 6553. The data sets and exposure times for the WFPC2 observations are listed in Table 2. An image of the WFPC2 chip 3 (core) is shown in Figure 1.

The images were calibrated using the standard HST pipeline procedure, and the long and short images were stacked separately using the IRAF routine CRREJ. To identify the stars in the image we ran DAOFIND with a $3 \sigma$ detection limit on the F814W images. There were no spatial offsets between the F555W and F814W images, so the F814W DAOFIND list of objects was also used for the F555W images. We carried out aperture photometry in F555W and $\mathrm{F} 814 \mathrm{~W}$, using an aperture radius of 2 pixels for all chips (PC: 0"09; WFC: 0".2). Our experience has been 
TABLE 1

BASIC PARAMETERS FOR NGC 6553

\begin{tabular}{|c|c|c|}
\hline Parameter & Value & Reference \\
\hline R.A. .. & 180917.6 & New measure, this paper \\
\hline Decl. .............. & -255431.3 & \\
\hline$(l, b)(\mathrm{J} 2000.0) \ldots . .$. & $5.2529,-3.0298$ & \\
\hline$[\mathrm{Fe} / \mathrm{H}] \ldots \ldots \ldots \ldots$. & -0.4 & Barbuy et al. 1999 \\
\hline$[\mathrm{Fe} / \mathrm{H}] \ldots \ldots \ldots \ldots$. & -0.1 & Carretta et al. 2001 \\
\hline$Z \ldots \ldots \ldots \ldots \ldots \ldots$ & $\approx Z_{\odot}$ & Barbuy et al. 1999 \\
\hline Age ............... & $12 \pm 2 \mathrm{Gyr}$ & Ortolani et al. 1995 \\
\hline$r_{c} \ldots \ldots \ldots \ldots \ldots \ldots$ & 0.55 & Harris 1996 \\
\hline$r_{h} \ldots \ldots \ldots \ldots \ldots \ldots$ & 1.55 & Harris 1996 \\
\hline$D_{\odot} \ldots \ldots \ldots \ldots \ldots \ldots$ & $\approx 4.4 \mathrm{kpc}$ & Derived \\
\hline$E(V-I) \ldots \ldots \ldots \ldots$ & 0.87 & Minimum, highly variable \\
\hline$E(B-V) \ldots \ldots \ldots \ldots$ & 0.72 & Minimum, highly variable \\
\hline$(m-M) \quad \ldots \ldots \ldots .$. & 13.2 & Suggested here \\
\hline$[\mathrm{Fe} / \mathrm{H}] \ldots \ldots \ldots \ldots$. & $\sim 0.0$ & Suggested here \\
\hline Age................ & $\sim 13 \mathrm{Gyr}$ & Suggested here \\
\hline
\end{tabular}

TABLE 2

WFPC2 DATA SETS FOR NGC 6553

\begin{tabular}{cccc}
\hline \hline Data Set & Filter & Exposure (s) & Date \\
\hline u4ax1301r ..... & F555W & 5 & 1998 Apr 24 \\
u4ax1302r ..... & F555W & 5 & 1998 Apr 24 \\
u4ax1303r ..... & F555W & 5 & 1998 Apr 24 \\
u4ax1304r ..... & F555W & 200 & 1998 Apr 24 \\
u4ax1305r..... & F555W & 200 & 1998 Apr 24 \\
u4ax1306r ..... & F555W & 200 & 1998 Apr 24 \\
u4ax1307r..... & F814W & 20 & 1998 Apr 24 \\
u4ax1308r ..... & F814W & 20 & 1998 Apr 24 \\
u4ax1309r ..... & F814W & 20 & 1998 Apr 24 \\
u4ax130ar ..... & F814W & 200 & 1998 Apr 24 \\
u4ax130br ..... & F814W & 200 & 1998 Apr 24 \\
u4ax130cr ..... & F814W & 200 & 1998 Apr 24 \\
\hline
\end{tabular}

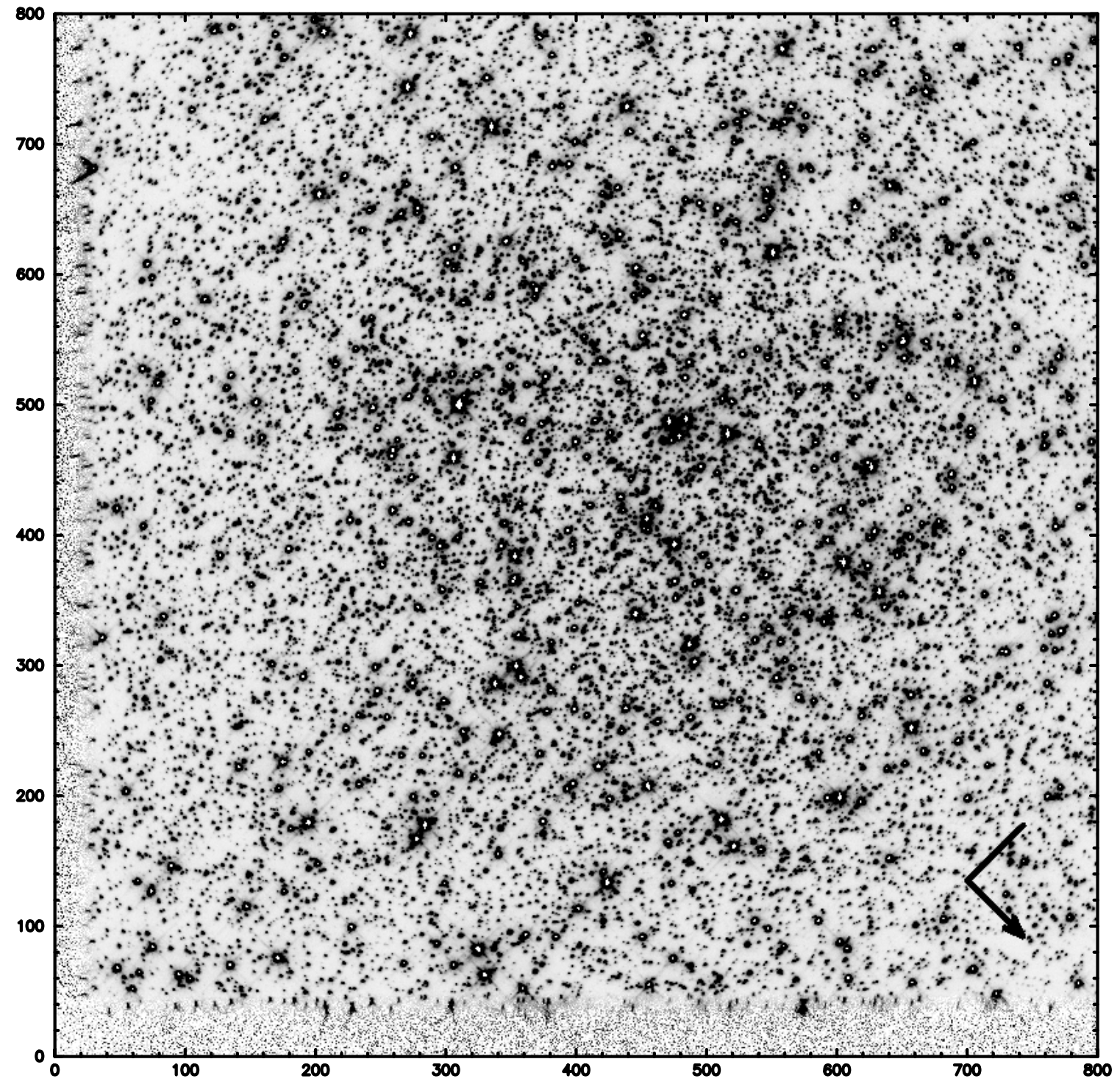

FIG. 1.-Visual image of NGC 6553. This image is from WFPC2 and shows chip 3 centered on the cluster core. The cluster appears essentially smooth and regular, even though patchy extinction may explain some of the properties of its color-magnitude diagram. The arrow indicates north, the line east. 
that aperture photometry, particularly on the undersampled WFC chips, produces tighter CMDs than PSF fitting. However, PSF fitting is useful for identifying and eliminating spurious detections via the ALLSTAR "sharpness" parameter. This parameter is defined as the difference between the square of the width of the object and the PSF. Therefore, we also carried out PSF fitting using the routine ALLSTAR, for which TinyTim PSFs were used. Note that the available STScI recipe for correction of the systematic photometric effects resulting from CCD CTE effects assumes 2 pixel radius aperture magnitudes.

The sharpness parameter as a function of F555W magnitude for the long exposures is plotted in Figure 2. The results are very similar for the short exposures and also for the F814W image. Selection limits were typically $-0.30<$ sharpness $<0.20$, but varied slightly from chip to chip. We also tried PSF fitting with a PSF constructed from stars in the image rather than from TinyTim. This did not result in significantly tighter sharpness distributions, and particularly for chip 3 , containing the cluster core, the field was sufficiently crowded that isolated stars suitable for constructing a PSF were rare. This crowding is responsible for the shallower limiting magnitude and the tilt in the distribution of points in Figure 2c, though the apparent increasing sharpness of stars in more crowded regions is somewhat
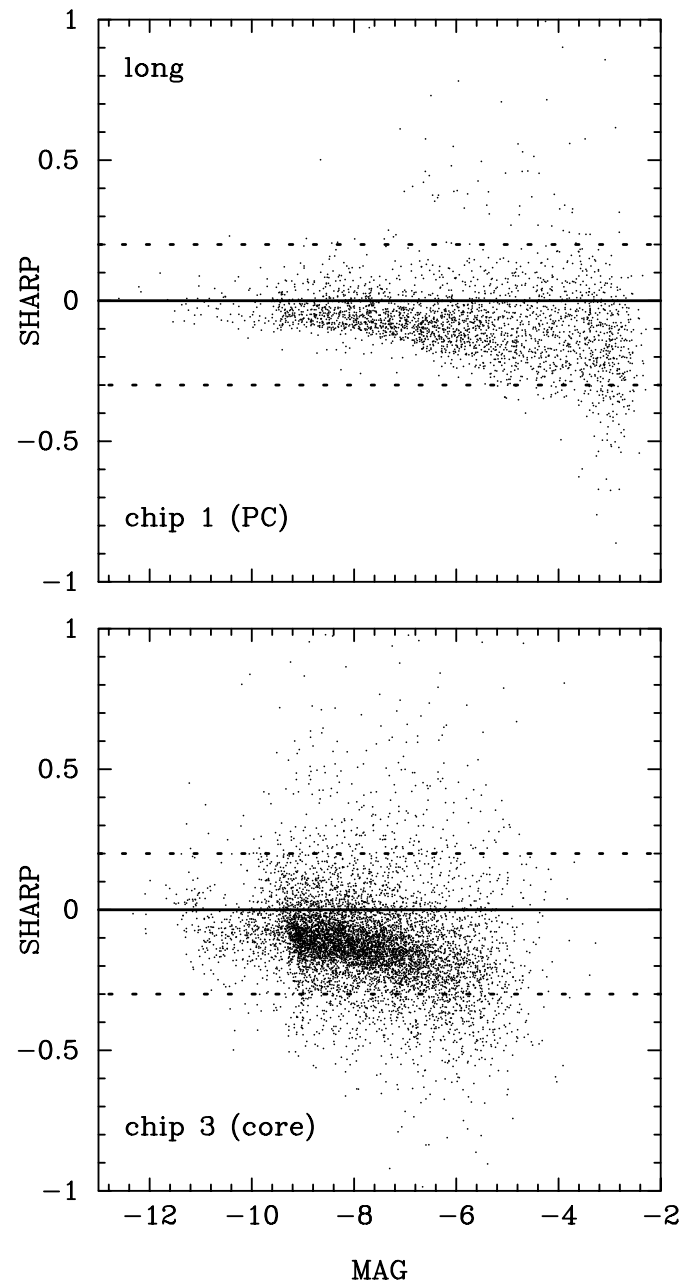

nonintuitive. We note, however, that the sharpness selection adopted is not critical for the purposes of this analysis.

Having removed spurious detections from both the F555W and F814W object lists, we then returned to the aperture photometry, with a 2 pixel aperture, and calibrated this following Baggett et al. (1997). The calibration includes corrections for charge transfer (in)efficiency (CTE; Whitmore, Heyer, \& Casertano 1999), geometric distortion (Holtzman et al. 1995), aperture corrections (a correction to a radius of 0.5 ) using bright stars in the image, and zero pointing from Baggett et al. (1997). Color-magnitude data for the separate chips are shown in Figures $3 a-3 d$ (short exposure) and Figures $4 a-4 d$ (long exposure).

Figures $5 a$ and $5 b$ show the completeness functions for $V$ and I, for both short (solid line) and long (dashed line) exposures. The difference between the short and long data is largest in the PC chip and smallest in the WFC chips, because of a better sampling of the PSF in the PC chip, making stellar detection more sensitive to exposure time. For the WFC chips, a stronger limitation in image classification will arise because of pixel subsampling, which reduces the ability to distinguish between extended sources and close pairs of stars. The net result is that, even at the bright end, some stars will be lost as they are merged with neighbors. This will also happen at the faint end, but there
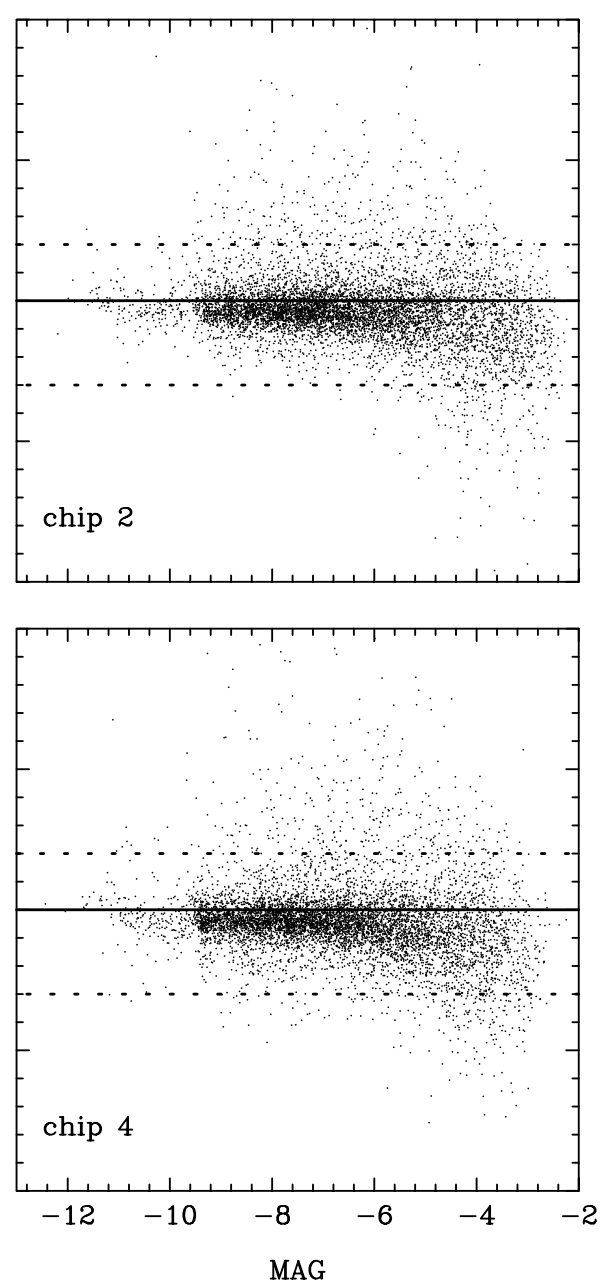

FIG. 2.- Sharpness parameter from PSF fitting (with TinyTim PSFs) as a function of magnitude for the long exposures taken with the F555W filter of NGC 6553 for the four WFPC2 chips. The adopted range of sharpness for real stars is between the dashed lines. Points falling above the top line are generally associated with diffraction spikes, while those lying below the bottom line are generally warm pixels or residual cosmic rays. The bottom left panel is for the cluster core (chip 3). Here crowding accounts for the fact that the points do not go as deep and for the trend in sharpness with magnitude. 

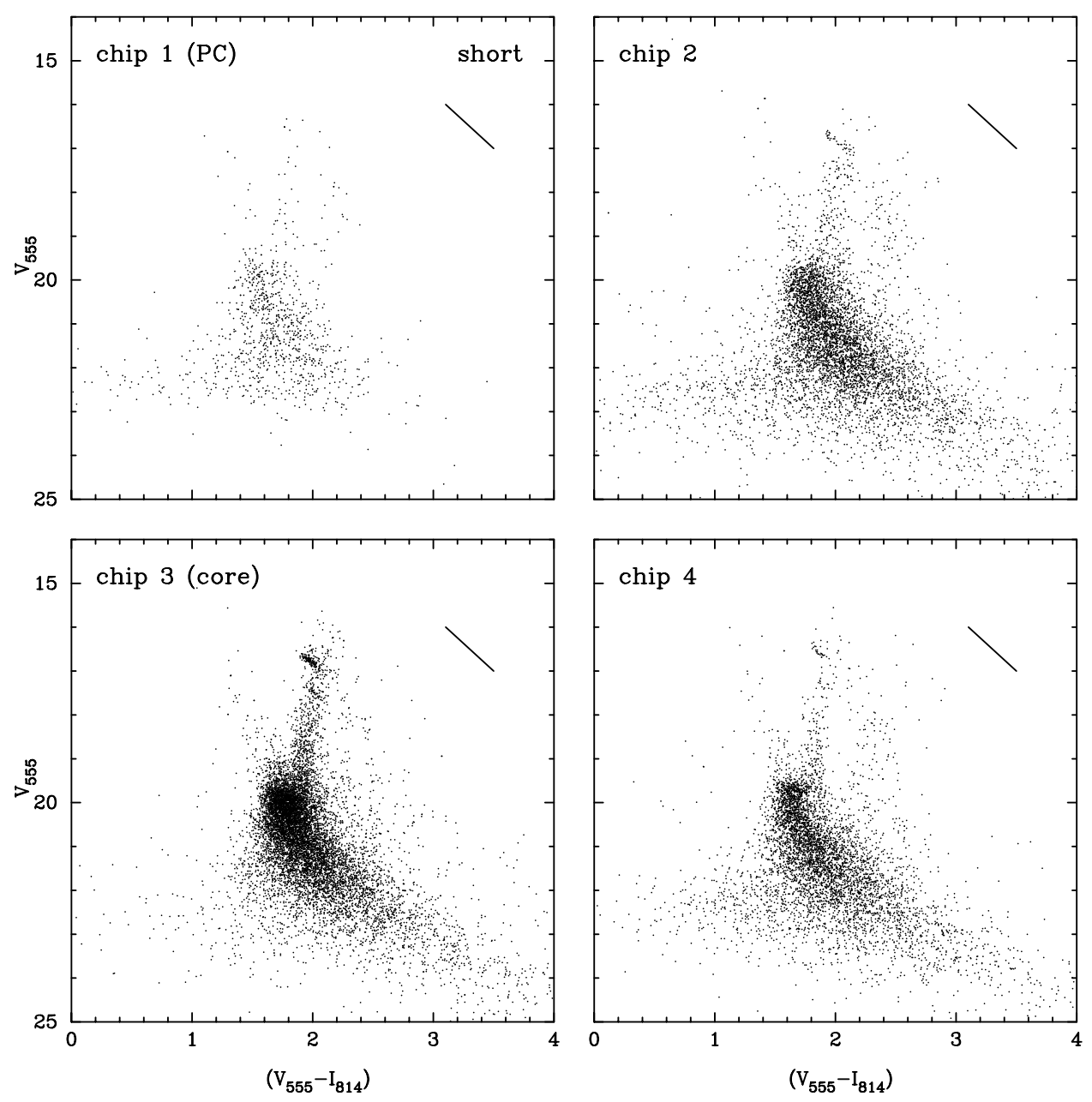

FIG. 3. - Color-magnitude data for the short exposures of NGC 6553. The length of the diagonal dash indicates the extinction in this field with the slope that of the reddening vector. The similarity of the slopes of the horizontal branch and of the reddening vector are evident. Brighter stars than those shown are saturated. The giant branch of the background bulge population is most clearly visible redward of the NGC 6553 giant branch in chips 2 and 4 .

stars will be lost mainly because of noise which begins to dominate. The effect is most evident in WF3 (cluster core), where the functions are the least sensitive to exposure time because completeness is strongly limited by crowding, not image depth. In general, the difference in the $V$ and $I$ exposure times (see Table 2) seems to indicate that, where crowding is most severe, there is not much gain in doing a long exposure.

The main features of the color-magnitude diagrams are discussed in detail below. These include the blue population brightward of the main-sequence turnoff, visible in the short-exposure data, the tilted horizontal branch (HB) and the clump below the HB, also visible in the short-exposure CMDs, and an apparent "second turnoff," an excess of stars near $(V, V-I)=(21,2)$. The second faint red turnoff is particularly prominent in chip WF4 (Fig. 4d).

\subsection{WFPC2: Results and Discussion \\ 2.1.1. Distance, Metallicity, Reddening}

There is only a moderate consensus on the values of distance, reddening, and metallicity for NGC 6553, derived from observations obtained from both the ground and space. From spectra of two cluster giants, Barbuy et al. (1999) derive a metallicity $[\mathrm{Fe} / \mathrm{H}]=-0.55 \pm 0.2$. Cohen et al. (1999), from high-dispersion Keck spectroscopy of five RHB stars deduce $[\mathrm{Fe} / \mathrm{H}] \approx-0.16$, revised by Carreta et al. (2001) upward to $[\mathrm{Fe} / \mathrm{H}] \approx-0.06$. Guarnieri et al. (1998) apply various methods of estimating metallicity based on the morphology of the giant branch both in $(K$, $J-K)$ and $(V, V-I)$ and adopt a mean value of $[\mathrm{Fe} /$ $\mathrm{H}]=-0.22 \pm 0.05$. The overabundance of several elements relative to $\mathrm{Fe}$ gives an overall abundance $Z \approx Z_{\odot}$ (Barbuy et al. 1999). Sagar et al. (1999) present a groundbased $(V-I)$ study of NGC 6553. From the difference in $V$ magnitude between the horizontal branch and the brightest point of the red giant branch (RGB), they estimate [Fe/ $\mathrm{H}]=-0.1$, although this value is uncertain because of the dramatic tilt of the $\mathrm{HB}$, which itself spans $0.5 \mathrm{mag}$, and the scarcity of stars at the brightest point of the RGB. Rather than trying to deduce a specific value for metallicity, we show below that the color-magnitude data favor a high metallicity, comparable to solar, in agreement with the more recent spectroscopic results.

Guarnieri et al. (1998) derive values of reddening and distance modulus for NGC 6553 of $E(V-I)=0.95$ and ( $m$ $-M)_{0}=13.6$. They comment that the largest source of uncertainty in the distance modulus is the ratio $A_{V} / E(B-V)$ that is needed to convert the reddening to an absorption (see Grebel \& Roberts 1995). Further discussion of all these studies is provided in Feltzing \& Gilmore (2000; note that there is a plotting error in the CMD of NGC 6553 in Feltzing \& Gilmore 2000, noted in a later erratum). 

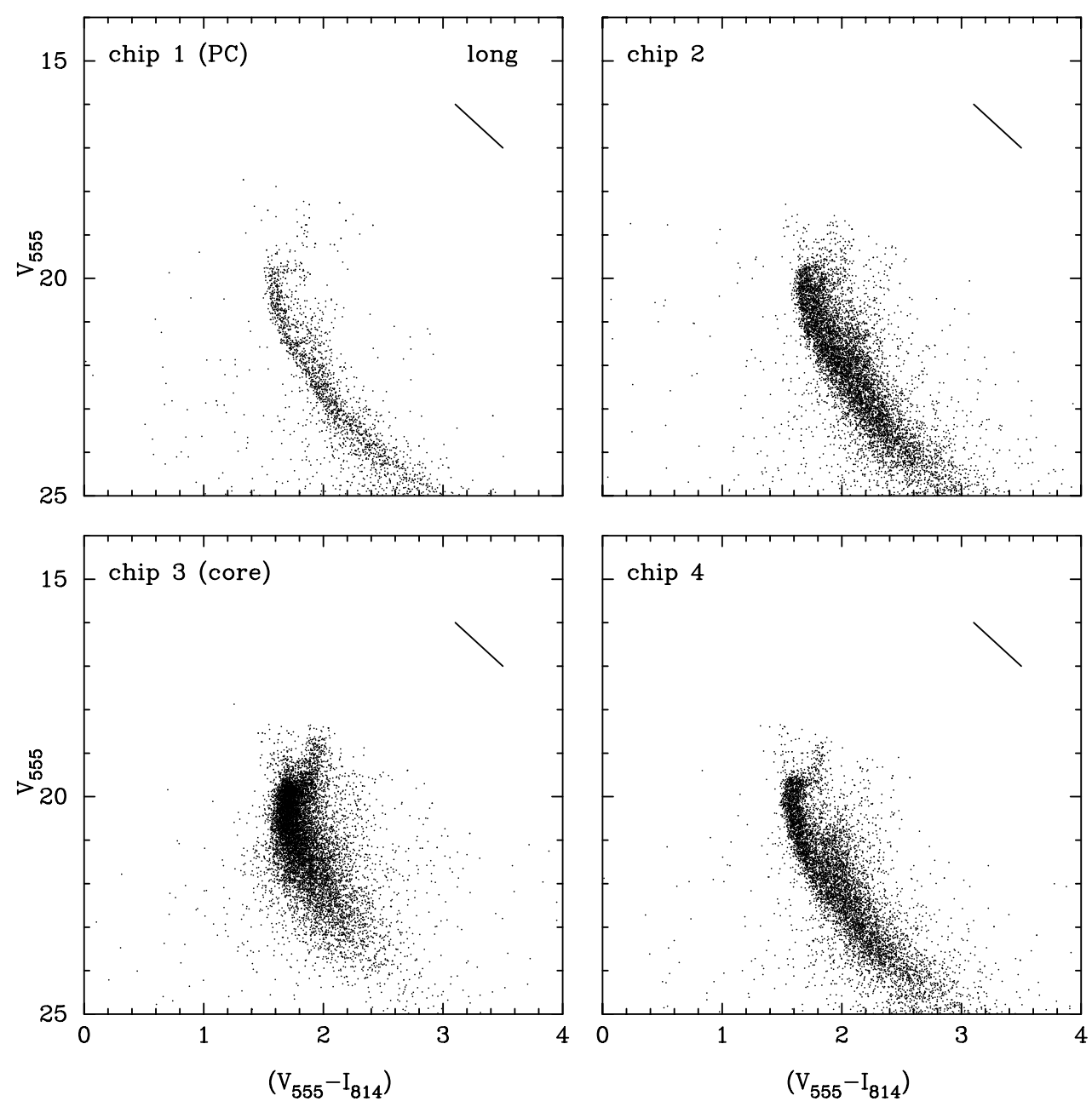

Fig. 4. - Color-magnitude data for the long exposures of NGC 6553. The diagonal line indicates the extinction, as in Fig. 3. Brighter stars than those shown are saturated. Note the presence of a red flare of stars near $(V, V-I)=(21,2)$, most clearly visible in chip 4 . We suggest this is the main-sequence turnoff of the background bulge population.

Figure 6 shows a composite CMD for NGC 6553 derived from the short exposures for all four chips. Photometric error bars are shown. A 12 Gyr isochrone is superposed. This is based on the Bertelli et al. (1994) isochrones, transformed to the HST filter system by G. Worthey (1998, private communication). The isochrone is for metallicity $[\mathrm{Fe} / \mathrm{H}]=-0.4$, reddening $E(B-V)=0.7$, and distance modulus $(m-M)_{0}=13.6$.

The same isochrone is superposed on the long-exposure data in Figure 7. The general shape of the isochrones, especially around the turnoff region, in spite of limitations with available metal-rich isochrones, suggests that NGC 6553 requires a higher metallicity, in agreement with the more recent spectroscopic studies. It is also clear that patchy extinction is affecting any conclusions. A possibility is that NGC 6553 is indeed more metal-rich than this isochrone, and than the bulge stars. We note that this possibility is consistent with the measured mean metallicity of the bulge, which peaks near $[\mathrm{Fe} / \mathrm{H}] \approx-0.3$ (see Fig. 2 of Wyse, Gilmore, \& Franx 1997), if NGC 6553 is indeed near solar metallicity and has enhanced $[\alpha / \mathrm{Fe}]$ values, as suggested by some studies.

To test this further we illustrate the scale of patchy extinction in Figure 8. This shows an enlargement of the main-sequence turnoff region, corrected for extinction using local values. For this correction, we fitted for reddening in $20^{\prime \prime} \times 20^{\prime \prime}$ subregions of the image: the good correlation between adjacent derived reddening values supports the validity of this approach, while the large scatter in some regions attests to patchy extinction on even smaller scales. As expected, the scatter around the turnoff is significantly reduced, and the isochrones fit significantly better. It does not, however, completely remove the tilt of the horizontal branch. Additionally, the apparent width of the main sequence is very much larger than typically seen in HST globular cluster studies, suggesting that residual extinction remain significant. We investigate this further in the next section.

We note that, in spite of residual uncertainty due largely to irreducible patchy extinction, the best fit distance we derive from this figure is $(m-M)_{0}=13.2,0.4 \mathrm{mag}$ shorter in distance modulus than previous estimates. This new value, together with other parameters for this cluster, is summarized in Table 1.

\subsubsection{Horizontal-Branch Morphology}

It has been noted by many previous authors, and is very obvious in Figure 6, that the horizontal branch in NGC 6553 is far from horizontal. Its range in $V$ spans $\sim 0.5 \mathrm{mag}$. Comparison with the direction of the reddening vector 

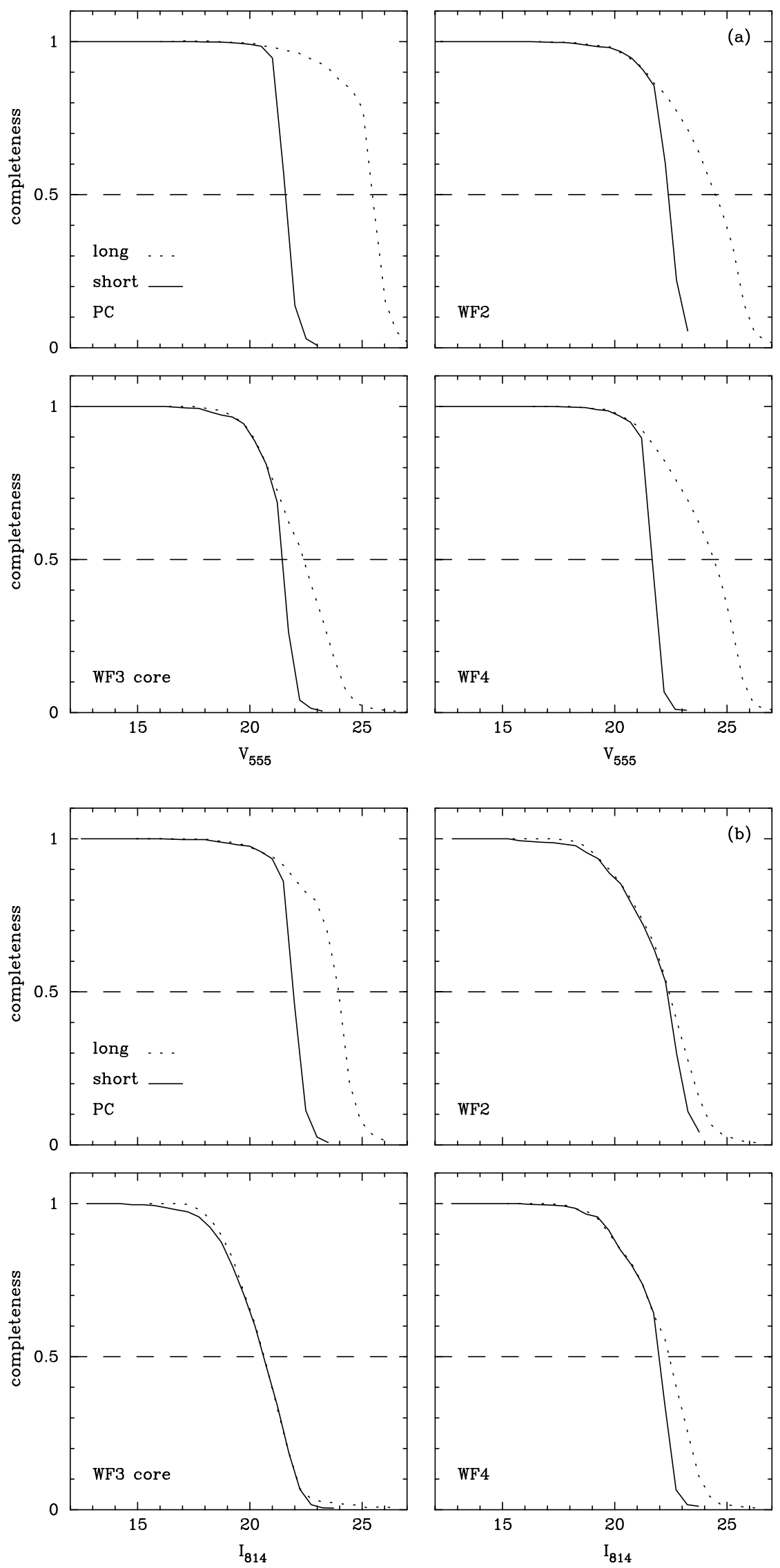

FIG. 5.- Completeness functions of NGC 6553 for $V_{555}(a)$ and $I_{814}(b)$ with short (solid line) and long (dashed line) exposures. The cluster's core is on the WF3 chip. The $V_{555}$ and $I_{814}$ exposure times can be found in Table 2 . 


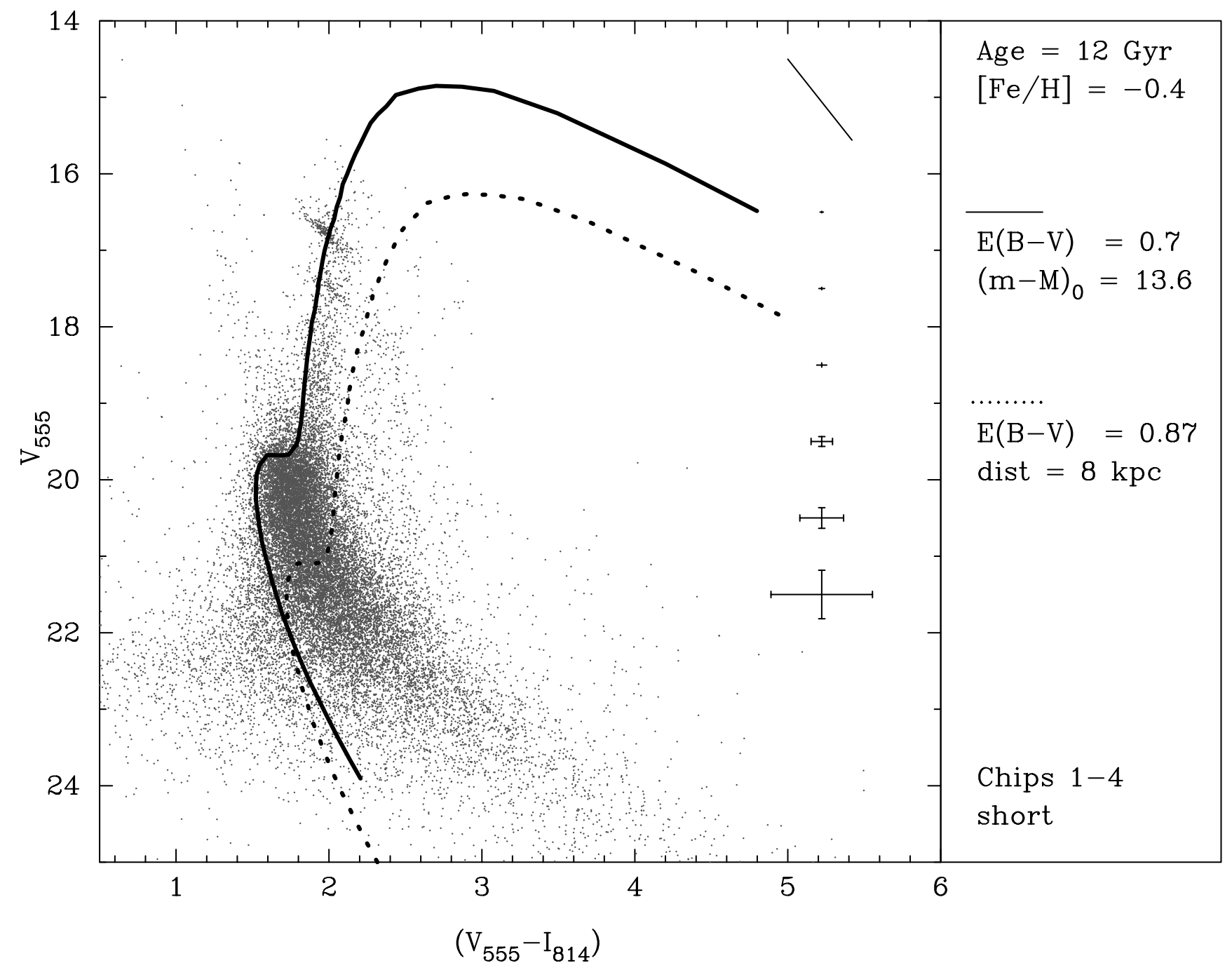

FIG. 6.-Combined color-magnitude data for the short-exposure observations of NGC 6553 from chips 1, 2, 3, and 4. A 12 Gyr isochrone for $[\mathrm{Fe} / \mathrm{H}]=-0.4$, reddening $E(B-V)=0.7$, and distance modulus $(m-M)_{0}=13.6$, values from the literature, is superposed. This isochrone is not a good fit to the main-sequence slope. The dashed isochrone represents the background bulge stars and has $[\mathrm{Fe} / \mathrm{H}]=-0.4, E(B-V)=0.87$ and distance $8 \mathrm{kpc}$. The diagonal line in the top right of the figure indicates the amount by which the isochrone has been offset to allow for reddening.

shown in Figure 6 shows that the orientation of the horizontal branch follows almost exactly the reddening slope in the CMD. Consequently, several authors have noted that much of this tilt might be attributable to differential reddening across the face of the cluster (see Barbuy, Bica, \& Ortolani 1998). Others suggest that metal line blanketing can produce a tilting of the $\mathrm{HB}$ at the high abundance of this cluster (see Ortolani, Barbuy, \& Bica 1990), so that both effects may contribute. We note below that the red flare of main-sequence stars near $(V, V-I)=(21,2)$, discussed further below, may also be caused by patchy extinction. The fainter red stars are apparent however some 1.5 mag fainter than the cluster main-sequence turnoff, while what would be the corresponding horizontal branch is apparent only $\sim 0.8 \mathrm{mag}$ fainter than the cluster HB, complicating any single explanation of both phenomena.

In order to investigate the simplest version of the differential extinction hypothesis, we show in Figure 9 the spatial distribution of blue and red HB stars in chip 3 (the cluster core). The full sample of $176 \mathrm{HB}$ stars was divided in half at the median color value $(V-I)=1.9$. There is a marginal tendency for the redder stars to be found preferentially in the top right of the distribution, suggestive of extinction effects. Nonetheless, the red-blue separation is not perfect, implying highly structured extinction; the dust must be patchy on scales $\lesssim 10^{\prime \prime}$.

To investigate this further we attempted to invert the extinction map, under the null hypothesis that all the HB slope is reddening. We assumed that the intrinsic horizontal branch is indeed horizontal in the $(V, V-I)$ plane. We then calculated the extinction required for each star independently, to move that star from its observed location in the CMD to lie on a dereddened, horizontal horizontal branch. This produced a set of extinction values, one per HB star. These were then used to define a two-dimensional map of extinction as a function of position across the face of the cluster. To reduce noise, this array was then smoothed with a fourth-order polynomial surface function, generating a two-dimensional extinction map. That map "predicts" a value of the extinction under this null hypothesis at every point on the cluster. We may then apply this map to the observed, uncorrected HB star photometry to see if this 


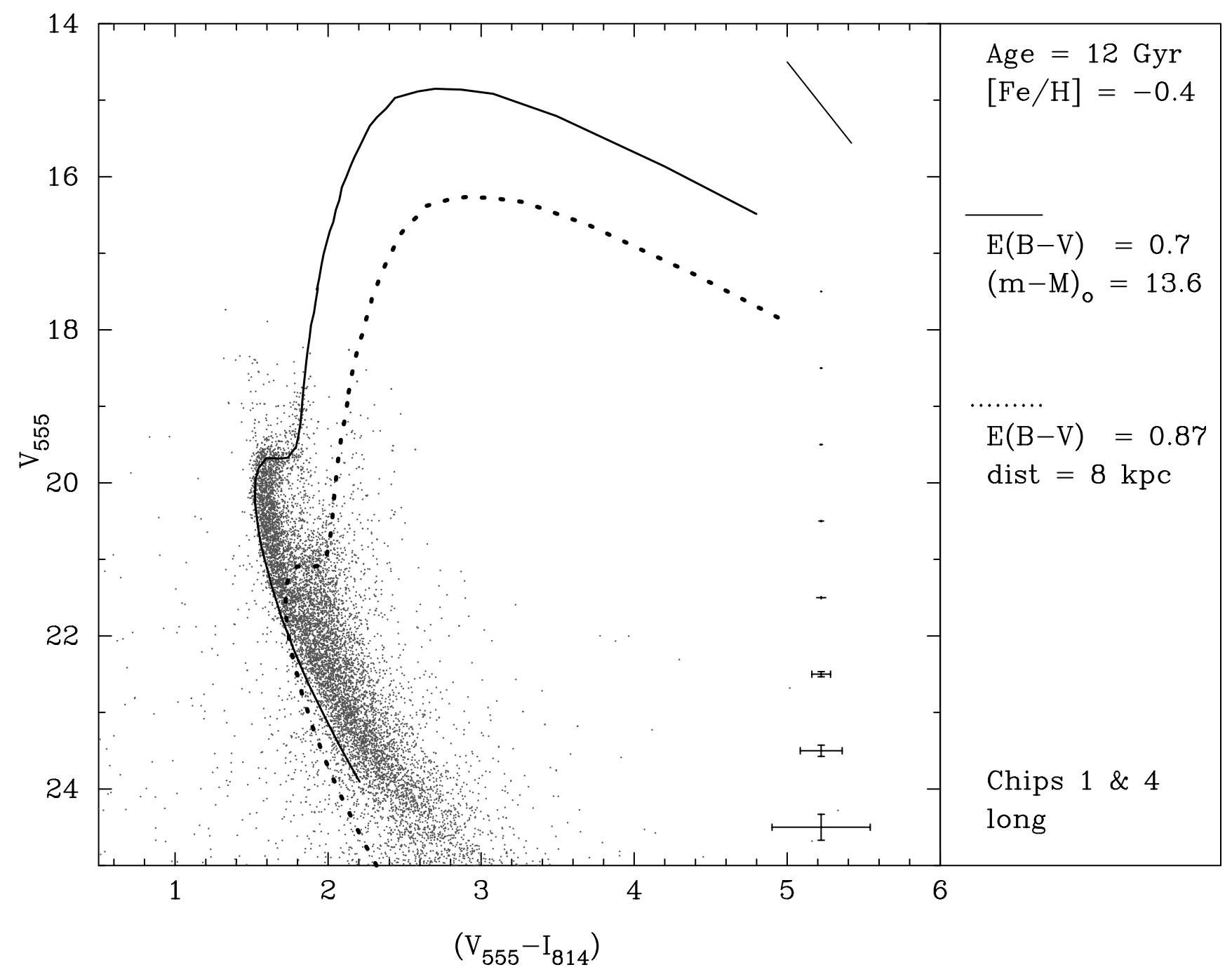

FIG. 7. - Combined color-magnitude data for the long-exposure observations of NGC 6553 from chips 1 and 4 . A $12 \mathrm{Gyr}$ isochrone for $[\mathrm{Fe} / \mathrm{H}]=-0.4$, reddening $E(B-V)=0.7$, and distance modulus $(m-M)_{0}=13.6$ is superposed, but does not represent the main-sequence slope well, as in Fig. 5. The dashed curve is a $12 \mathrm{Gyr}$ isochrone with the same metallicity for a distance of $8.0 \mathrm{kpc}$, with reddening $E(B-V)=0.87$, expected for a background bulge population.

smoothed extinction map has any validity: if the null hypothesis is valid, a nearly horizontal HB should result, and in addition the scatter in the RGB and near the mainsequence turnoff should be significantly reduced.

Figure 10 shows the outcome of this attempt to correct for differential reddening. This figure applies to stars in the WF3 chip (core of NGC 6553), and shows the photometry before (top panel) and after (middle panel) correction by the derived reddening map, defined by a polynomial surface fit of order 4. The reduction in photometric scatter, and HB tilt, is at best minimal. There is similarly only a marginal reduction of scatter in the main-sequence turnoff data, with this reddening map. Our attempts to derive a higher spatial resolution differential extinction map assuming the stars in the "red flare" are really cluster main-sequence turnoff stars also failed. In Figure 10 (bottom panel, we show the HB data from Figure 8. This allows the reader to make a direct comparison of both methods used to attempt to correct for differential reddening.

We thus conclude, along with Ortolani et al. (1990) and Sagar et al. (1999), that differential reddening cannot explain all the tilt of the horizontal branch in NGC 6553, though it probably contributes. We return to the implications for the explanation of the red flare stars below.

\subsubsection{Blue Straggler Stars and Core Structure}

\subsubsection{New Center Estimate}

The available estimation of the center of NGC 6553 is that of Harris (1996). We have calculated an improved center, from the distribution of stars near the mainsequence turnoff. This value is given in Table 2 . The new surface density profiles for stars above the turnoff, at the turnoff, and below the turnoff, are illustrated in Figure 11. The local dip in the counts at zero for the turnoff and main-sequence stars is suggestive of some incompleteness in the very crowded central regions, even at the corresponding bright magnitudes. As the distribution of RGB stars shows, there is a small group of very bright giants at the cluster center, complicating faint photometry.

The short-exposure CMDs in Figure 6 contain a number of blue stars brighter than the MSTO, which may either be blue stragglers or foreground stars belonging to the Galactic disk population. Most globular clusters contain blue 


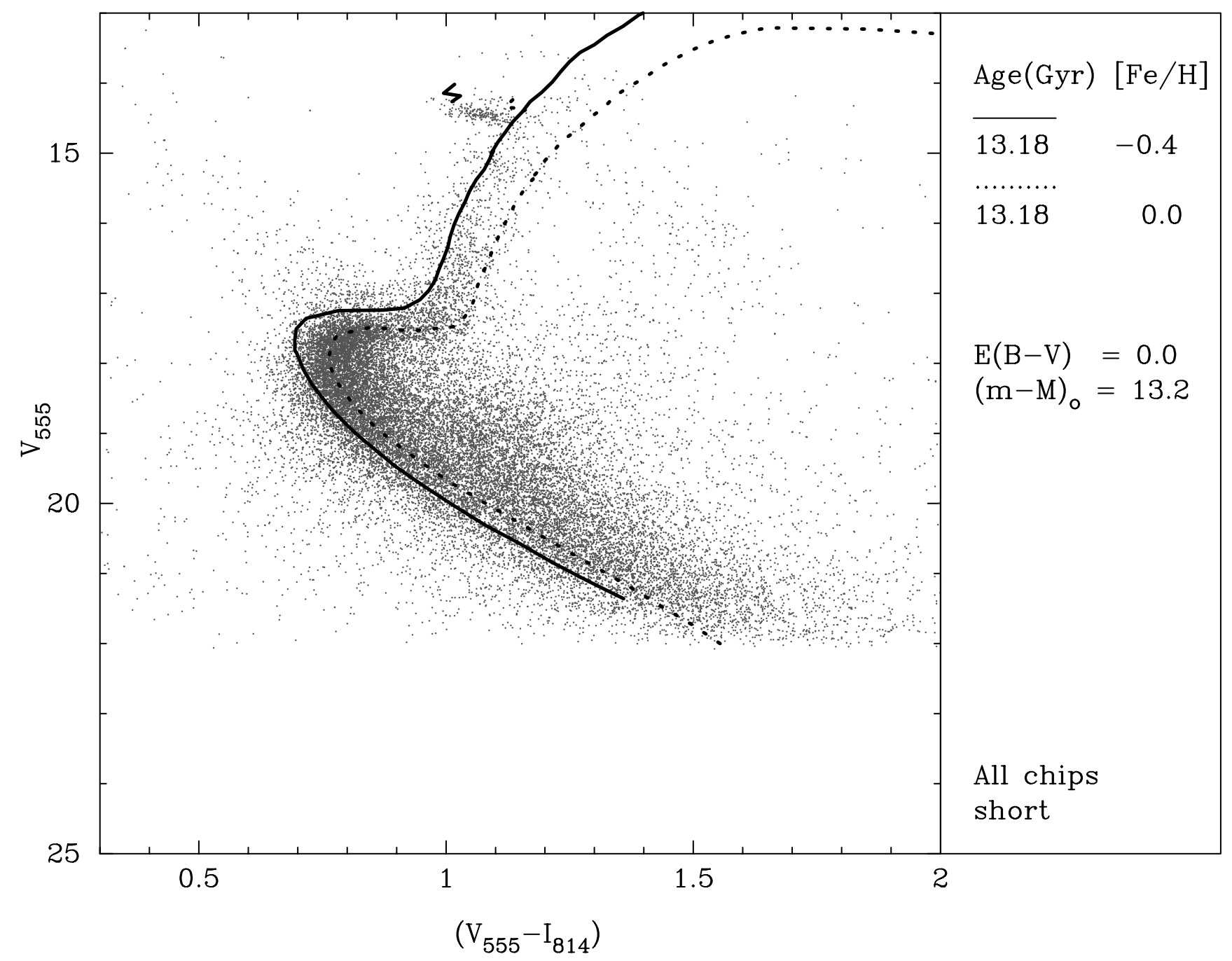

FIG. 8. - Color-magnitude data corrected for reddening in $20^{\prime \prime} \times 20^{\prime \prime}$ regions. The overlaid isochrones are for $[\mathrm{Fe} / \mathrm{H}]=0.0$ and -0.4 , and age $13.18 \mathrm{Gyr}$. The fit around the turnoff and subgiant branch is clearly improved relative to use of a single-extinction value. The deduced distance modulus is 0.4 mag smaller than that derived in previous studies.

stragglers, while recent studies of four globular clusters show these stars to be strongly concentrated toward the cluster centers (Ferraro et al. 1997; Mandushev et al. 1997; Guhathakurta et al. 1998; Rey et al. 1998). Very recently, Zoccali et al. (2001) present proper motion data showing the candidate blue straggler stars in the central regions of the cluster are indeed members.

To investigate the radial distribution of the blue straggler candidates in NGC 6553, we have isolated on each chip stars with $V_{555}<19.2$ and $1.04<\left(V_{555}-I_{814}\right)<1.74$. The numbers of stars on each of Chips 1 to 4 are 16, 52,121, and 62 , respectively. Given that chip 1 covers an area 4 times smaller than the other chips, the surface densities are roughly equal for chips 1,2 , and 4 . Chip 3 , containing the cluster core, contains twice as many blue straggler candidates.

Figure 12 shows the radial surface density profile of the blue stragglers, normalized by the surface density of mainsequence stars with $19.6 \leq V \leq 20.1$. This magnitude range was chosen as both the stellar mass and the number count completeness for the main-sequence stars and blue stragglers is similar, so this ratio is robust. The profile is centered on WF3 $(471,478)$.
The true surface density of blue stragglers peaks at the origin in this coordinate system. The main-sequence star counts plot is very flat (Fig. 11) and has, if anything, a slight dip at the coordinate center $(471,478)$, which may be due to crowding-induced incompleteness at the origin. If so, this may slightly overstate the ratio of blue stragglers to mainsequence stars at the origin, but as a $10 \%-20 \%$ uncertainty. Small number statistics are important here. There are only seven blue straggler stars in the innermost bin; at the first maximum spike in the plot there are only six blue stragglers, though the second spike is robust, with 12 blue stragglers in the annulus beyond 3 core radii. That is, there are relative maxima in the radial surface density distribution of blue stragglers at both small and large radii.

The blue straggler radial distribution in another cluster, M3 (Ferraro et al. 1997) shows a similar anomalous distribution, and has been modeled by Sigurdsson et al. (1994) under the assumption that the main mechanism for producing blue stragglers is collisions between primordial binaries and single stars. In this model, recoil during collisions displaces blue stragglers from the core on formation and produces an initial $r^{-2}$ radial distribution out to some radius determined by the depth of the cluster potential. Blue strag- 


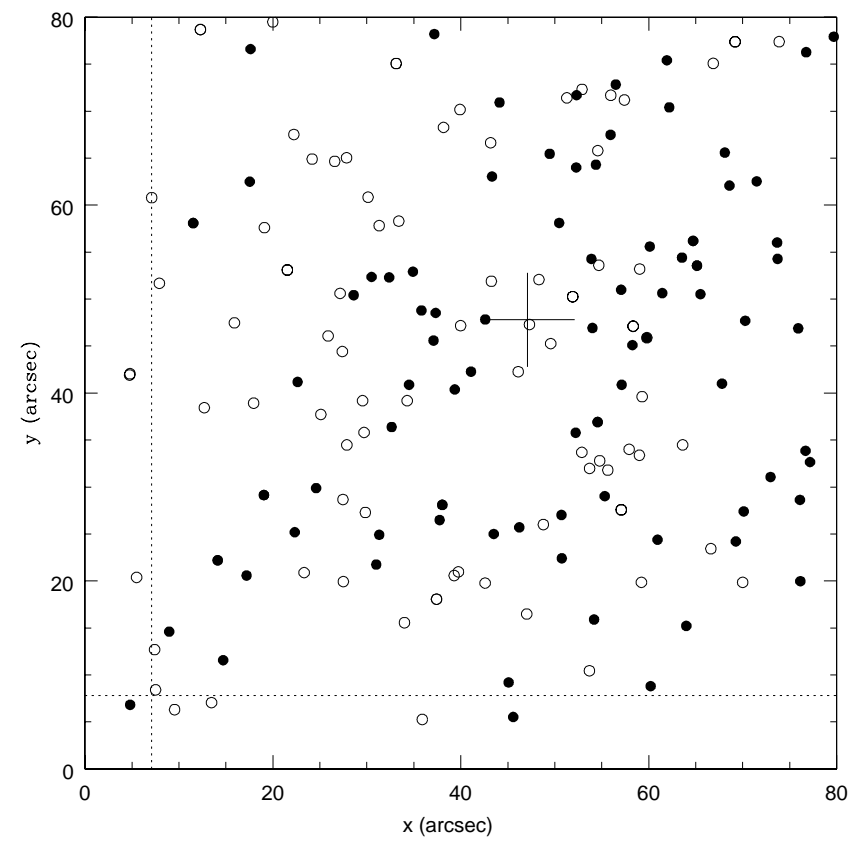

FIG. 9.- Spatial distributions of the 176 horizontal stars in the cluster core to test patchy extinction as an explanation of the tilt of the horizontal branch. We divided the stars into two groups: those bluer than $V-I=1.9$ (open circles) and those redder (filled circles). The dotted area is centered on the cluster center which is indicated with the cross at $(471,478)$. Some blue-red segregation is apparent, indicating that differential extinction has at least contributed to the morphology of the horizontal branch. However, the lack of a clear segregation suggests that another effect is also relevant.

glers have higher mean mass than the typical mainsequence star in the cluster, so dynamical friction then brings the blue stragglers back into the cluster core. The blue straggler distribution is thus enhanced in the core, and depleted out to some critical radius $r_{b}$ where the dynamical friction timescale becomes comparable to the blue straggler lifetime. Assuming a dispersion $\sigma \sim 7 \mathrm{~km} \mathrm{~s}^{-1}$ (Cohen et al. 1999) and a core density, $\rho_{0}=10^{5} M_{\odot} \mathrm{pc}^{-3}$, we expect substantial depletion of blue stragglers.

The density distribution of blue stragglers rises inside the core radius as the blue stragglers have a higher mean mass than the mean mass of the core. The cluster core radius here is $30^{\prime \prime}$, so the blue stragglers would be expected to form a dynamical subcore with radius of about $20^{\prime \prime}$. The density of the blue stragglers should rise as $\sim r^{-2}$ between $20^{\prime \prime}$ and $30^{\prime \prime}$, and thus the surface density should $\propto r^{-1}$. Outside the core, going from about a core radius to about the half-mass radius, the density distribution of blue stragglers should be steeper than $r^{-2}$ in the region where dynamical friction efficiently transports the stars to the core, and then the density flattens out again near the (projected) radius where the dynamical friction timescale becomed comparable to the blue straggler lifetime. Outside this radius we predict the surface density distribution of blue stragglers should decline again, but less steeply than the density of the main-sequence stars.

\subsubsection{Red Giant Branch Luminosity Function}

The luminosity function of the red giant branch is a test of stellar evolutionary models, and in particular it provides a consistency test of models of old metal-rich stars. Figure 13 shows an enlargement of the red giant branch region of the short-exposure CMD for all four chips and the corre-
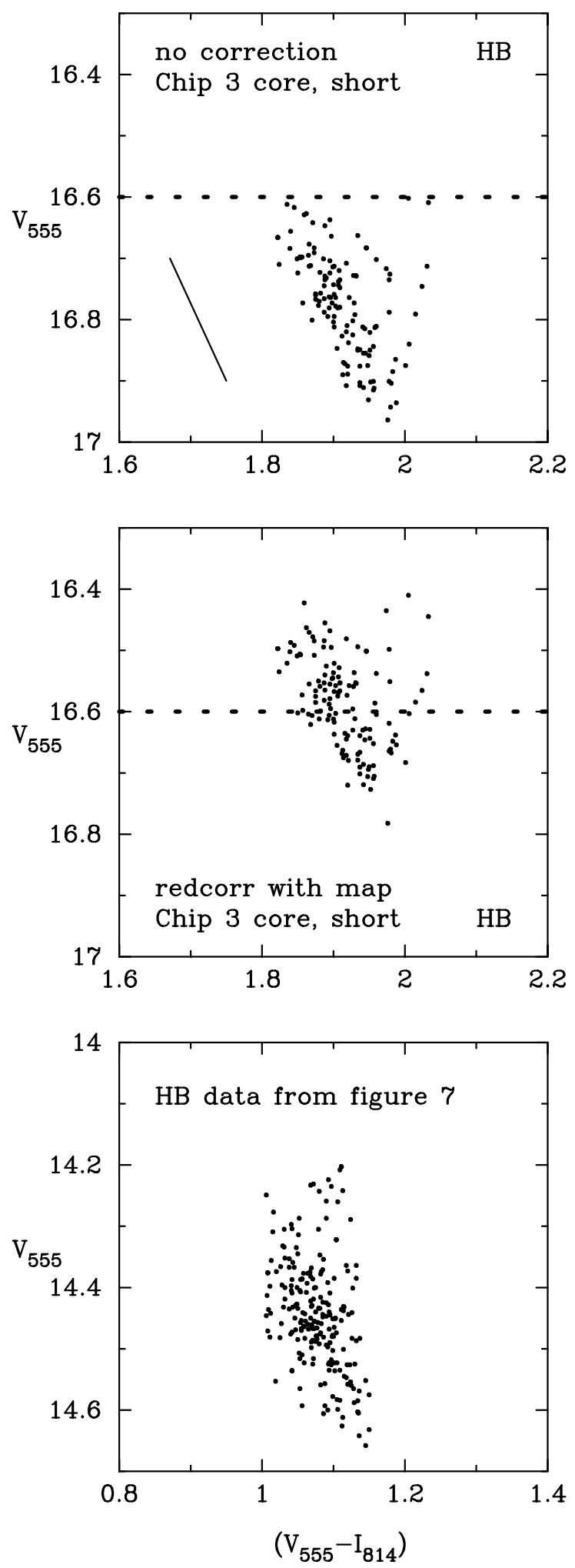

FIG. 10.-Top: Enlargement of the color-magnitude data for the horizontal branch stars from Fig. 3. The diagonal line is the reddening vector, which is seen to have a similar, but not identical, slope to that of the horizontal branch. Middle: Same stars after derivation and application of a two-dimensional extinction map, derived assuming the horizontal branch is indeed horizontal, and then smoothed in spatial distribution. The dashed line indicates the assumed location of this horizontal branch. This assumption is clearly not supported by the extinction-corrected data, showing that much of the morphology of the horizontal branch is intrinsic to the stars and not an artifact of variable extinction on spatial scales of several arcseconds or larger. Finally, the bottom panel shows the HB data from Fig. 7, illustrating the residual astrophysical contribution to the tilt of the HB. 


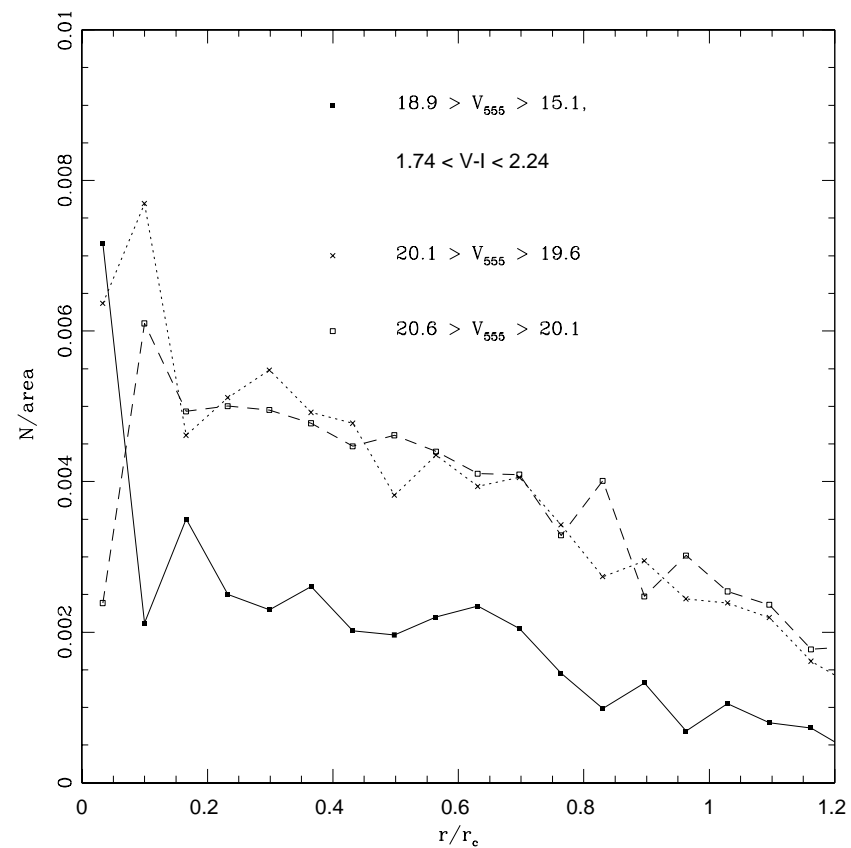

FIG. 11.- Radial surface density profiles for the three different luminosity groups of stars indicated. The brightest group is the RGB, the second group stars near the main-sequence turnoff, and the third group stars just below the turnoff. The core radius is $\sim 30^{\prime \prime}$.

sponding luminosity function. The horizontal branch shows up prominently, peaking at $V_{555}=16.7$. A second peak is clearly visible $\sim 0.9 \mathrm{mag}$ below the HB and may even be tilted parallel to the horizontal branch, indicative of patchy extinction as an explanation. The second peak has been discussed, for example, by Lanteri Cravet et al. (1997) and by Sagar et al. (1999), and it has been attributed to a phase of stellar evolution where the star loops in its ascent of the

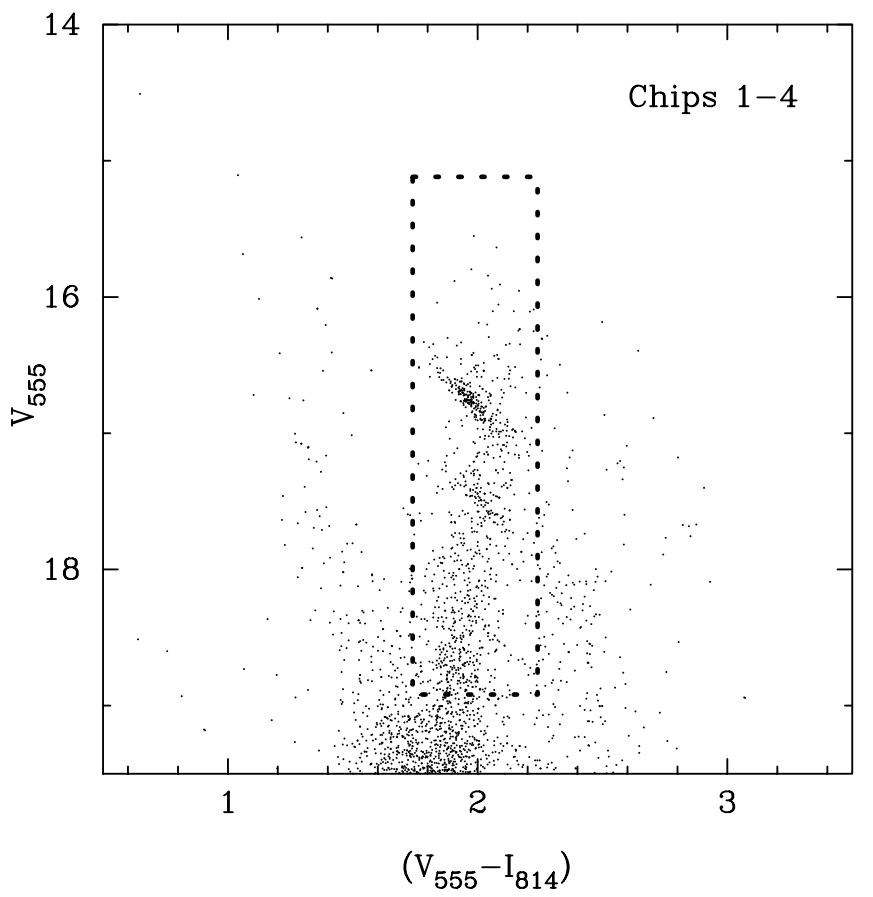

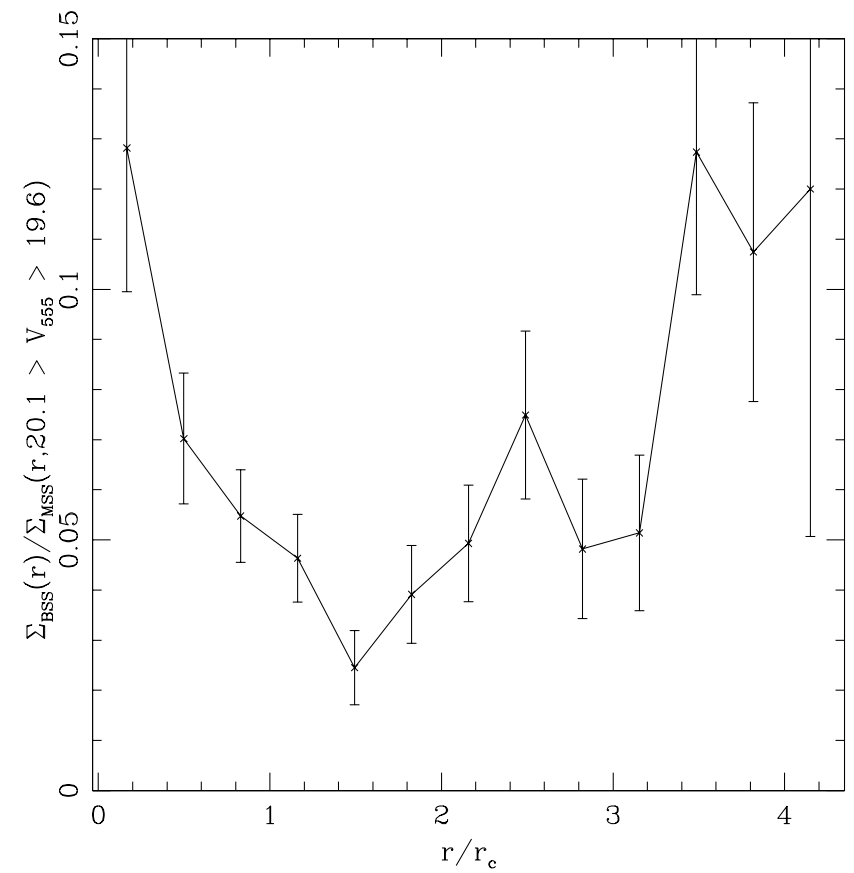

FIG. 12.-Ratio of the surface density of blue straggler to mainsequence turnoff stars in NGC 6553, in the apparent magnitude range is indicated in the caption. There is a relative excess of blue stragglers at both small and large distances from the cluster center.

RGB. Deferring to those analyses, we note only that the number of stars in this "RGB bump" remains poorly determined. Figure 13 suggests some $30 \%$, significantly larger than the $19 \%$ of Lanteri Cravet et al. and the $17 \%$ of Sagar et al.

Another suggestion is that the RGB bump may be confused by superposition on the cluster RGB of background

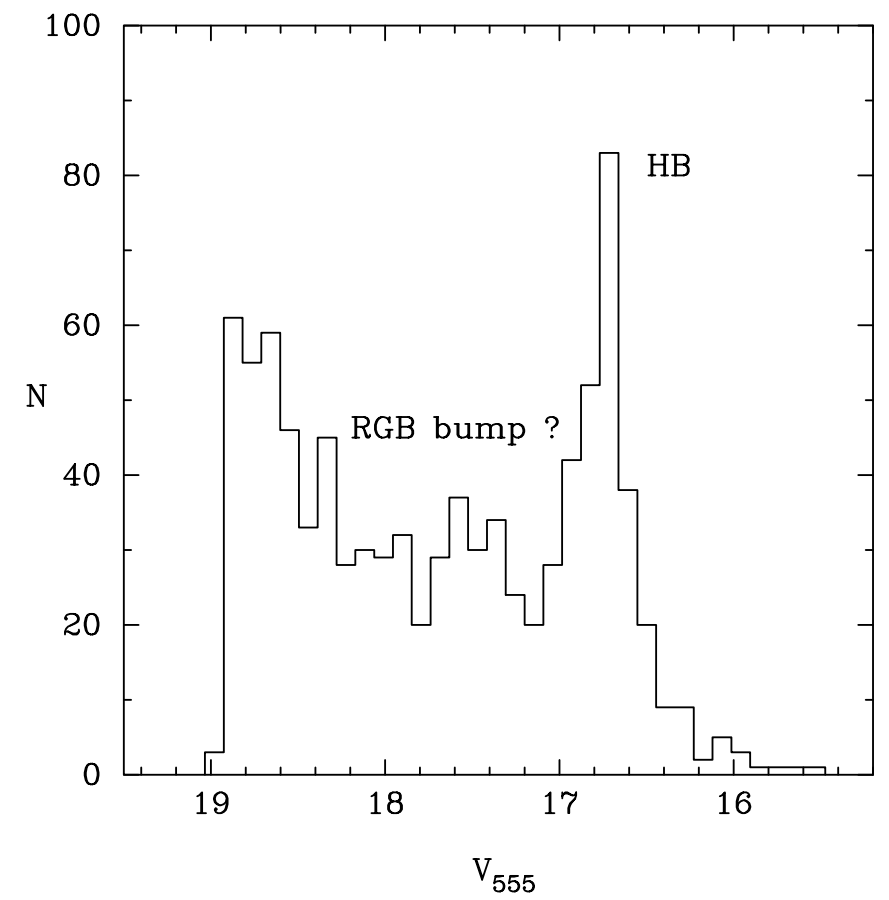

FIG. 13.-Enlargement of the red giant branch region of the color-magnitude diagram from Fig. 3 (left panel). The sloping horizontal branch is apparent, as is the concentration of stars some one magnitude fainter. The area outlined by the box is that used for derivation of the giant branch luminosity function (right panel). The luminosity function maxima due to the horizontal branch and RGB bump are clearly visible. 
field HB stars, associated with the second turnoff discussed below. In this case, the slope cannot be attributed to common differential extinction, since the background would have to suffer less extinction than the foreground cluster. A further problem is that the "second bump" has the same color $(V-I \approx 2)$ as does the second turnoff: in general, one expects a metal-rich old population to have a horizontal branch that is redder than the main-sequence turnoff color, as shown by the isochrone in Figure 6. A stellar evolutionary explanation of the observed RGB bump seems the most likely.

\subsubsection{The Red Flare}

The color-magnitude data shown here and in earlier published studies of this cluster indicate a substantially broader main-sequence width than is typical for HST data, with the scatter in color increasing substantially fainter than $V=21$. Indeed, the longer exposure color-magnitude data show an asymmetric color distribution about the mainsequence ridge line, especially visible as a redward extension of the data near $(V, V-I)=(21,2)$ in chips 1 and 4 (Figs. $7 a$, $7 d$ ). This may be equally described as an apparent second turnoff, implying a background population, or as a red flare, implying a part of the NGC 6553 stellar population that is substantially more reddened than the average.

Can the excess be due to extinction? Casual inspection of Figure 7 shows that this faint red group of stars lies in the CMD in a direction close to that of the reddening vector, relative to the cluster turnoff. Could these faint red stars then be cluster members, obscured by up to an additional $E(B-V) \approx 0.7 \mathrm{mag}$ ? Patchy extinction is also a plausible explanation for the morphology of the horizontal branch in this cluster. As described and investigated above, the slope of the HB is very similar to the slope of the reddening vector. We note that, as is also shown above for the HB, since this extra red population is not spatially highly segregated from stars with apparently much smaller reddening, this equivalently implies either extinction inside the cluster itself, or patchy extinction on very small spatial scales. This possibility is investigated in detail above, in the discussion of the horizontal branch morphology, and shown not to be viable.

We may test directly if this feature corresponds to a background population, such as the turnoff of the inner Galactic bulge. The most important conclusion is that it is not possible to fit the fainter turnoff with the same isochrone as for NGC 6553, simply shifted to a larger distance. A larger distance modulus and a smaller value of reddening are required. If the bulk of the bulge stars are at a distance of $8.0 \mathrm{kpc}$, then the reddening required to fit the turnoff is $E(B-V)=0.87$. This fit is illustrated by the dotted lines in Figures 6 and 7. The required extinction in this case is less than that for the cluster, although that is some $3 \mathrm{kpc}$ closer to us. Might there be a patch of extinction covering just the cluster, with lower extinction adjacent? This is difficult to arrange, as the background bulge stars, if that is what they are, are seen through the cluster, rather than off to one side of it.

In principle, a bluer isochrone, that is a more metal-poor population, at the distance of the bulge, and with extinction comparable to that in the foreground, is feasible. Thus, the red flare is consistent with being the main-sequence turnoff of the background bulge field stars, at a distance of $8 \mathrm{kpc}$, but only if the bulge field population is more metal-poor than is NGC 6553. There are some stars, visible in the short-exposure CMDs in Figures $3 a-3 d$, which might be considered members of a corresponding giant branch, but too few to help in the present analysis. They may support a future comparative spectroscopic abundance analysis with the members of NGC 6553.

\subsubsection{Conclusions from the WFPC2 Data}

We have tested the hypothesis that all the complexities of the observed optical color-magnitude data, especially the tilted horizontal branch and the existence of the red flare, are an artifact of very patchy extinction. We exclude this hypothesis above. This then requires an astrophysical explanation for the slope of the horizontal branch, with high cluster metallicity being the most obvious possibility. This is also consistent with the observed cluster RGB bump in the luminosity function, as noted by earlier workers. What then is the red flare? The best available explanation is that it is the background bulge turnoff field stars viewed through the cluster. In this case, the bulge field stars are not consistent with the NGC 6553 CMD simply shifted to larger distance: the bulge cannot have the same metallicity as does the cluster, but must be more metal-poor. This conclusion is consistent with published spectroscopic data for the bulge if the cluster abundance is indeed near solar. That is, NGC 6553 is not a simple template match to the mean Galactic bulge star, being probably more metal-rich.

Nonetheless, all conclusions on the age, distance, and metallicity of NGC 6553, and on the nature and/or reality of the red flare that depend sensitively on photometry are clearly suspect, given the extreme and patchy extinction.

\section{NGC 6553: STIS PHOTOMETRY}

Our STIS image of NGC 6553 is centered at R.A. $(\mathrm{J} 2000.0)=18^{\mathrm{h}} 09^{\mathrm{m}} 11^{\mathrm{s}}$, decl. $(\mathrm{J} 2000.0)=-25^{\circ} 54^{\prime} 33^{\prime \prime}$, about $1^{\prime}$ from the cluster center, and it overlaps with the PC images described above. Three images were taken with exposure times 30, 300, and $2046 \mathrm{~s}$. The data sets are listed in Table 3. An image of the field is shown in Figure 14. In imaging mode, the STIS detector has a size of $28^{\prime \prime} \times 51^{\prime \prime}$ and a scale of 0.05 pixel $^{-1}$. We used the STIS long-pass filter $\left(I_{\mathrm{LP}}\right)$, which essentially spans Johnson-Cousins $V, R$, and $I$. We discuss photometric transformations of this passband further below, but show that the STIS bandpass is very similar to the $I$-band for globular cluster stars. Instrumental parameters and a summary of in-orbit performance of STIS can be found in Sahu et al. (1999).

The exposures were calibrated using the standard pipeline (CALSTIS), and stacked using the task DRIZZLE. The resulting summed image was run through our own photometry pipeline, which consists of identifying stars, fitting them with PSFs constructed from isolated stars in each image, and discarding objects with anomalous values of the ALLSTAR sharpness parameter, as for the WFPC2 data. The sharpness parameter, as a function of magnitude, is

TABLE 3

STIS DATA SETS FOR NGC 6553

\begin{tabular}{ccc}
\hline \hline Data Set & Exposure (s) & Date \\
\hline o4ax14010..... & 30 & 1998 Mar 5 \\
o4ax14020..... & 300 & 1998 Mar 5 \\
o4ax14030..... & 2046 & 1998 Mar 5 \\
\hline
\end{tabular}




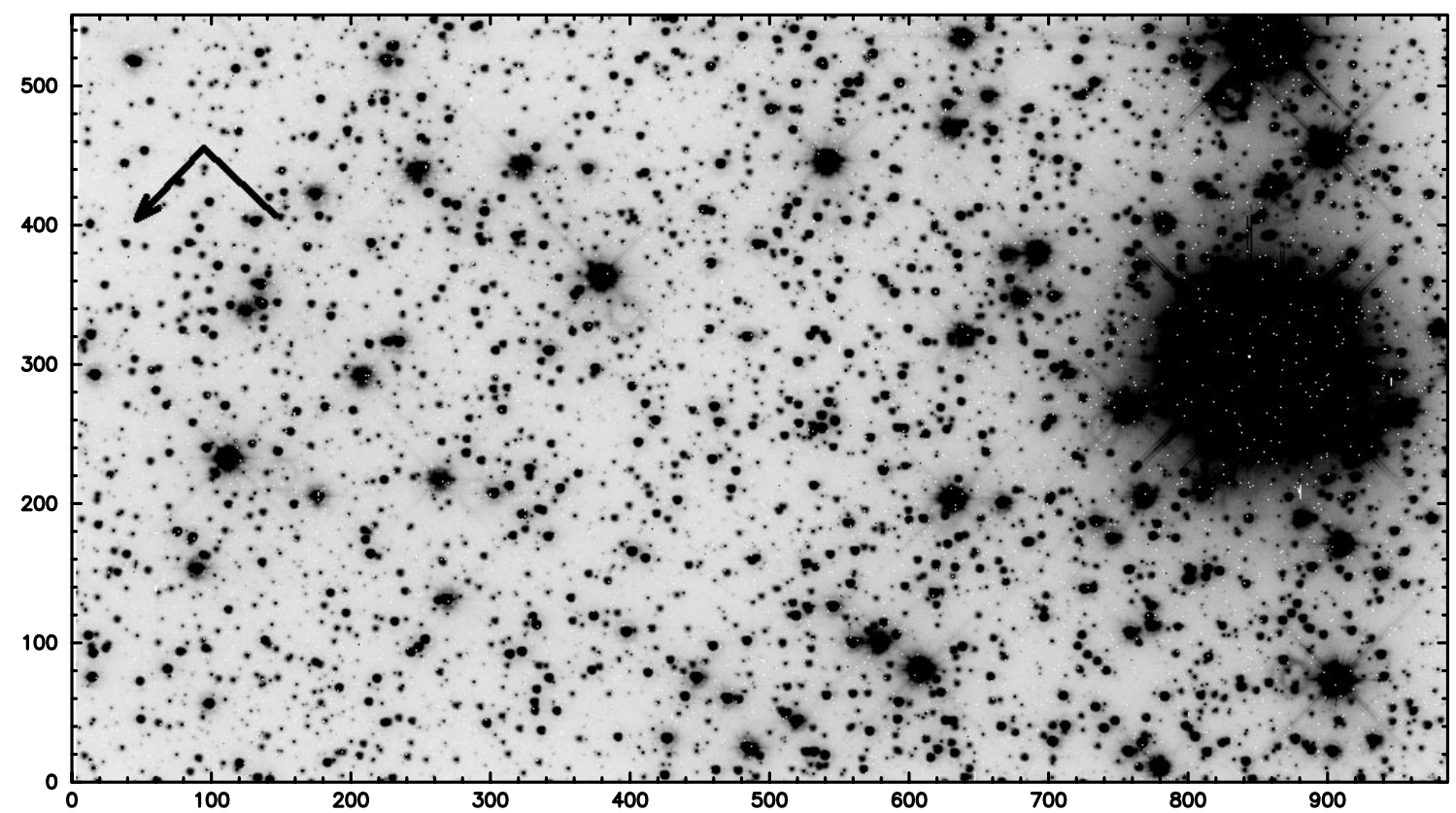

FIG. 14. - STIS image of NGC 6553. The image has size $29^{\prime \prime} \times 58^{\prime \prime}$. The faintest stars visible have magnitudes $M_{I} \sim 24$. The arrow indicates north, the line east.

plotted in Figure 15, which may be compared with the corresponding figure for the WFPC2 data in Figure 2. Our selection limits of $-0.10<$ sharpness $<0.15$ are indicated. There is a clear separation between point sources and detections that are slightly resolved (above the top line), which fall mainly along diffraction spikes and in the wings of saturated stars. Our final magnitudes are derived from 2 pixel radius aperture photometry. We make no correction for STIS CTE, since there is no significant correction at

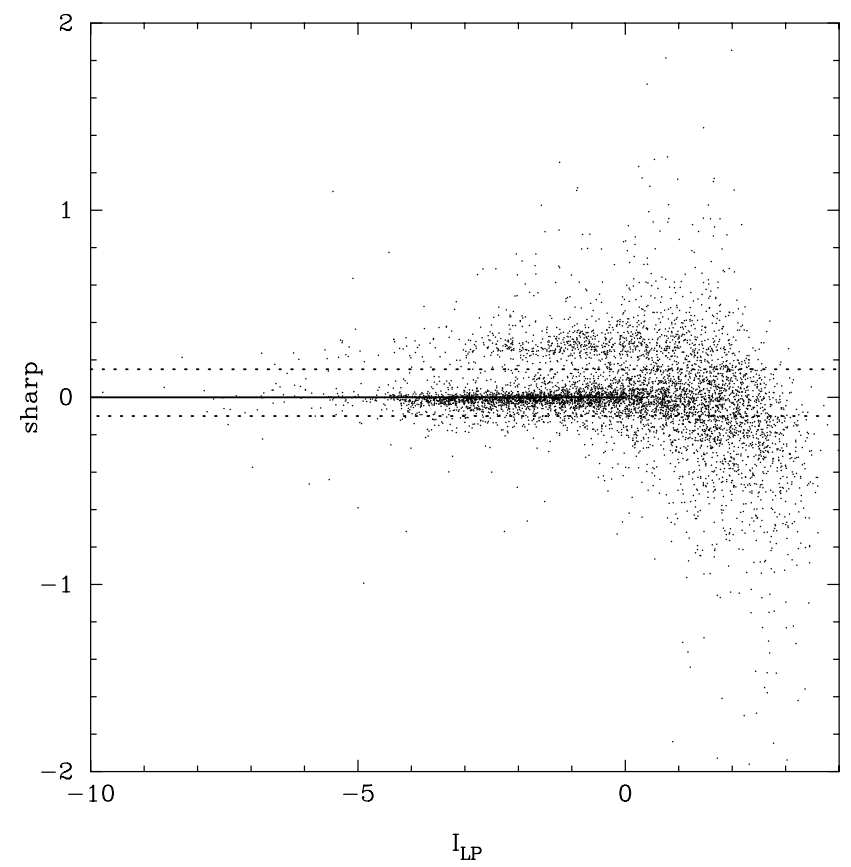

FIG. 15.- Sharpness parameter from PSF fitting plotted as a function of magnitude for STIS photometry of NGC 6553. Points selected as stars fall between the dotted lines. Points falling above the top line are generally associated with diffractions spikes, while those lying below the bottom line are generally warm pixels.
GAIN $=4$ appropriate for our data (Gilliland, Goudfrooij, \& Kimble 1999).

Our pipeline also determines completeness as a function of magnitude from a grid of 209 artifical stars added to the stacked image, and recovered along with the real stars. Completeness as a function of magnitude is shown in Figure 16.

\subsection{A STIS Luminosity Function for NGC 6553}

Figure 17 shows the STIS luminosity function for NGC 6553 , both raw and corrected for incompleteness. The corresponding luminosity function for 47 Tuc is overplotted for

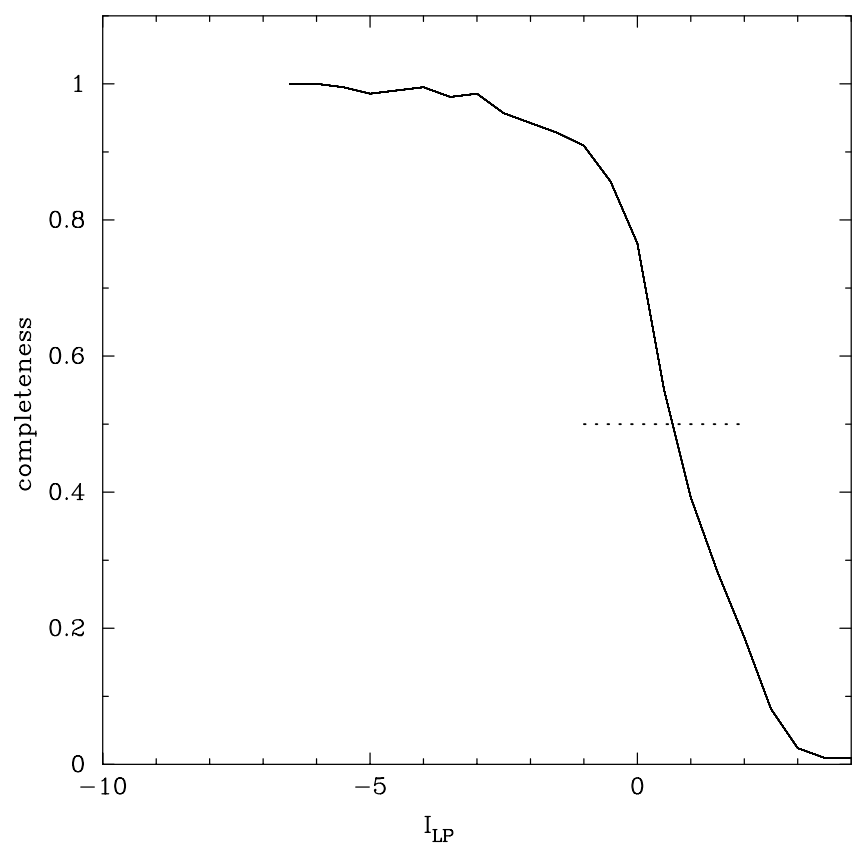

FIG. 16.-Completeness as a function of STIS magnitude for NGC 6553. The horizontal line indicates the $50 \%$ completeness limit. 

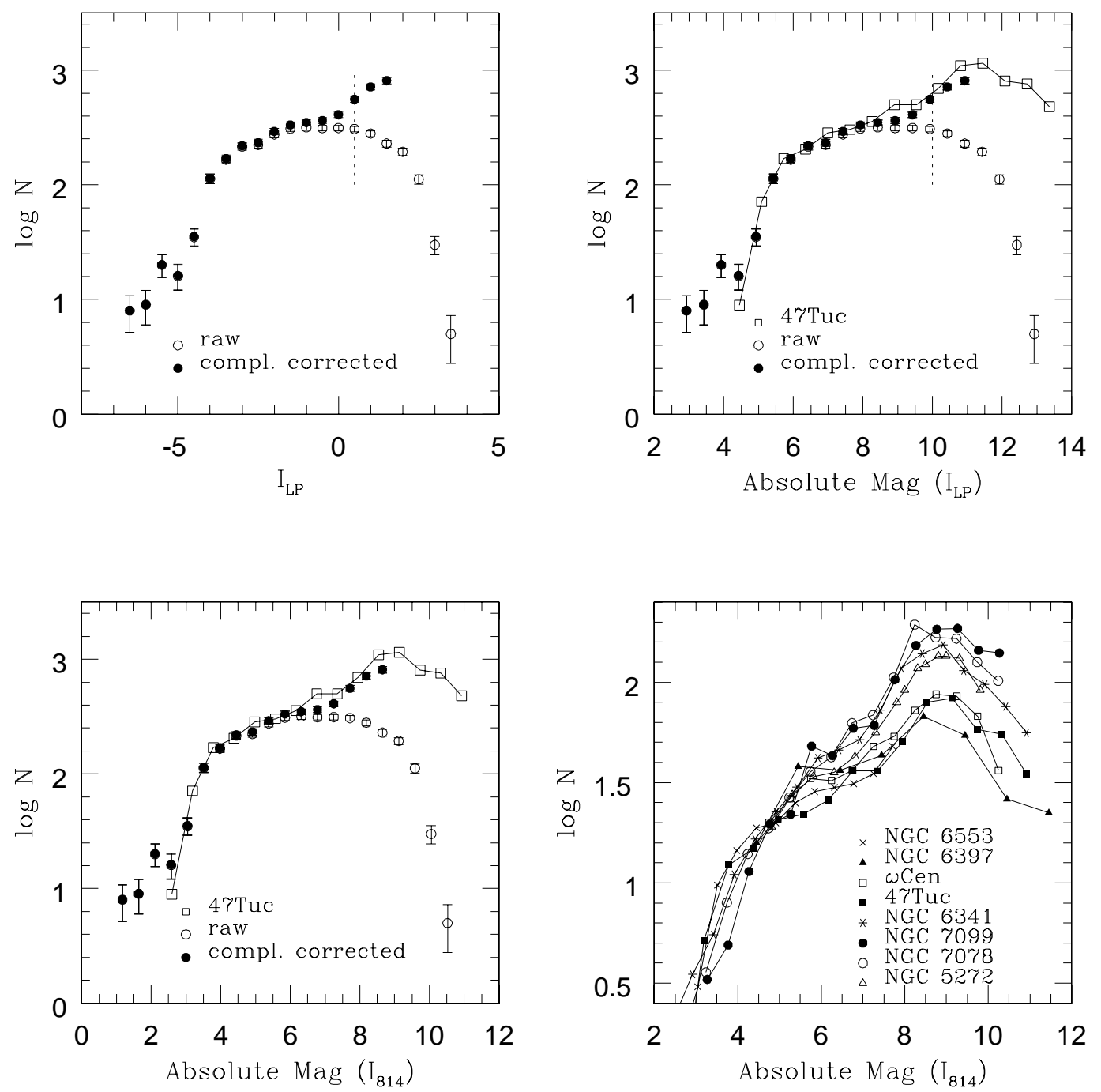

FIG. 17.-STIS and $I$-band luminosity functions, for a field $\sim 1^{\prime}$ from the center of NGC 6553 . The top left panel shows the raw and completenesscorrected luminosity function in the STIS instrumental system. The top right shows the same function converted to absolute STIS magnitude after reddening and distance modulus correction. In both of these the vertical dashed line indicates the $50 \%$ completeness limit. The bottom left panel shows the luminosity function for NGC 6553 after conversion of the STIS absolute magnitudes into absolute $I_{814}$ magnitudes, using the transformation of this paper. The luminosity function of $47 \mathrm{Tuc}$ is also shown for comparison. The bottom right panel shows absolute $I_{814}$ luminosity functions for seven globular clusters, including NGC 6553.

comparison. We have also converted the STIS measured instrumental magnitude to an absolute magnitude in the STIS $I_{\mathrm{LP}}$ passband. We do this converting from our utilized aperture radius of 2 pixels $\left(00^{\prime \prime} 1\right)$, with our measured aperture correction of $-0.5 \mathrm{mag}$ to an aperture correction radius at 0 ". 5 , a photometric zero point in $\mathrm{AB}$ magnitudes of $\mathrm{ZP} \mathrm{P}_{\mathrm{AB}}=$ 25.28 (Gardner et al. 2000), a reddening of $E(B-V)=0.7$, and an absolute distance modulus of $(m-M)_{0}=13.6$. The reddening and distance information are from Guarnieri et al. (1998). The value of $E(B-V)$ has been transformed to an absorption in the STIS $I_{\mathrm{LP}}$ passband by $A_{I_{\mathrm{LP}}}=2.505 E$ $(B-V)$ by integration across the passband. We also transform the STIS instrumental magnitude into an absolute magnitude in the $I_{814}$ passband, using our own STIS-LP versus WFPC2 relation derived below (see $\S 5.1$ ). This facilitates comparison with other published HST cluster luminosity functions and isochrones.

In Figure 17 we show also a comparison of seven available $H S T$ globular cluster luminosity functions, including now our transformed data for NGC 6553 and other data from the literature (Elson et al. 1995; Santiago. Elson, \& Gilmore 1996; Piotto, Cool, \& King 1997; de Marchi 1999). While the luminosity functions agree well at brighter magnitudes, the faint ends differ markedly. Such differences have been noted before, and have been attributed to metallicity effects, evaporation of low-mass stars, relative binary fractions, and stripping of low-mass stars by tidal shocking (see von Hippel et al. 1996; Piotto et al. 1997; Piotto \& Zoccali 1999; Elson et al. 1999; Paresce \& de Marchi 2000). All these effects must apply at some level and will have different levels of importance in different clusters. The metallicity dependence of the mass-luminosity relation will provide a systematic change in luminosity function shape with abundance (von Hippel et al. 1996). Mass differences and differences in core parameters will ensure that different clusters are in a different state of dynamical evolution toward eventual evaporation. Location in the Galaxy will induce different tidal stripping effects.

While full discussion of the relative importance of these effects is beyond the present paper, we do recall the result of 
Elson et al. (1999), who showed there is a rather good correlation between the shape of the cluster luminosity function, as shown in Figure 17, and the distance of the cluster from the Galactic plane. This latter quantity of course correlates strongly with the external tidal field. The final panel of Figure 17 is an updated version of the corresponding figure from Elson et al. (1999). Further analyses of these important and interesting effects are underway, using the latest GRAPE N-body calculations and our HST data (Aarseth 1999).

\section{NGC 6553: NICMOS PHOTOMETRY}

Our NICMOS NIC2 field is located at R.A. $(\mathrm{J} 2000.0)=18^{\mathrm{h}} 09^{\mathrm{m}} 11^{\mathrm{s}}$, decl. $(\mathrm{J} 2000.0)=-25^{\circ} 54^{\prime} 33^{\prime \prime}$. These coordinates overlap the region covered by the PC. The scale of the NIC2 camera is 0.0757 pixel $^{-1}$, giving a field of view of 19 ".4 on a side. We have obtained two exposures of 160 and $514 \mathrm{~s}$ in $\mathrm{F} 110 \mathrm{~W}(\approx J)$ and two exposures of 576 and $1026 \mathrm{~s}$ in $\mathrm{F} 160 \mathrm{~W}(\approx H)$ in MULTIACCUM mode. Instrumental parameters and in-orbit characteristics of NICMOS can be found in Dickinson et al. (1999). Details of the data sets can be found in Table 4. An image of the NICMOS F110W field is shown in Figure 18.

The images were recalibrated using the standard pipeline

TABLE 4

NICMOS NIC2 DATA SETS FOR NGC 6553

\begin{tabular}{cccc}
\hline \hline Data Set & Filter & Exposure (s) & Date \\
\hline n4ax12hcq ...... & F110W & 160 & 1998 Mar 2 \\
n4ax12hiq ...... & F110W & 514 & 1998 Mar 2 \\
n4ax12h5q..... & F160W & 576 & 1998 Mar 2 \\
n4ax12heq...... & F160W & 1026 & 1998 Mar 2 \\
\hline
\end{tabular}

CALNICA, and stacked using the task MSCOMBINE. Aperture photometry was performed following the proposed NICMOS recipe and photometric calibration cookbook. ${ }^{3}$ We used the IRAF/DAOPHOT task PHOT with an aperture radius of 0.15 and an aperture correction for a radius of 0 ". 5 . The aperture corrections were obtained using seven stars on each image. The mean value of the aperture corrections with their associated $\sigma$ are $J_{110}:(1.505,0.032)$ and $H_{160}:(1.678,0.032)$. A correction to a nominal infinite aperture was performed by multiplying the measured count rate of each star by 1.15 . We then converted the count rates into flux, and then into magnitudes $\left(J_{110}\right.$ and $\left.H_{160}\right)$ using the photometric keywords PHOTFNU and ZPVEGA obtained from the NICMOS Handbook (Dickinson et al. 1999). Table 5 lists the values used in this paper. Care was taken in order to mask out the coronagraphic hole region (radius $\approx 7$ pixels at the position near $[184,44]$ as it can be misidentified for a star in the IRAF/DAOPHOT routines.

Figure 19 shows the near-IR NICMOS color-magnitude diagrams, $[J,(J-H)]$ and $[H,(J-H)]$ derived from the

\footnotetext{
${ }^{3}$ See www.stsci.edu/instruments/nicmos and the NICMOS data handbook available there.
}

TABLE 5

NICMOS NIC2 PHOTOMETRIC KeYwordS

\begin{tabular}{ccc}
\hline \hline Filter & $\begin{array}{c}\text { PHOTFNU } \\
\left(\text { Jy DN }^{-1}\right)\end{array}$ & $\begin{array}{c}\text { ZPVEGA } \\
(\mathrm{Jy})\end{array}$ \\
\hline F110W ....... & $2.288 \mathrm{E}-6$ & 1773.7 \\
F160W ...... & $2.337 \mathrm{E}-6$ & 1039.3 \\
\hline
\end{tabular}

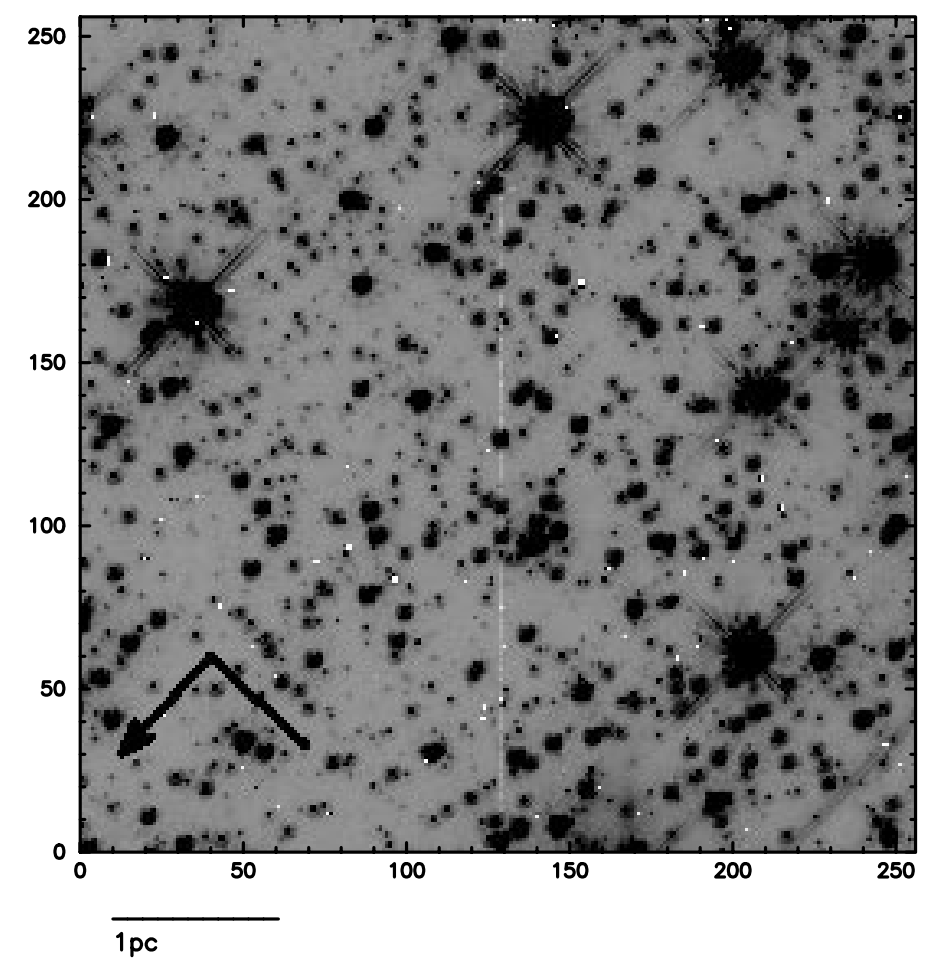

FIG. 18.-NICMOS NIC2 $J$-band (F110W) image of NGC 6553. The arrow indicates north, the line east, and the spatial scale at the cluster is indicated by the scale bar. 

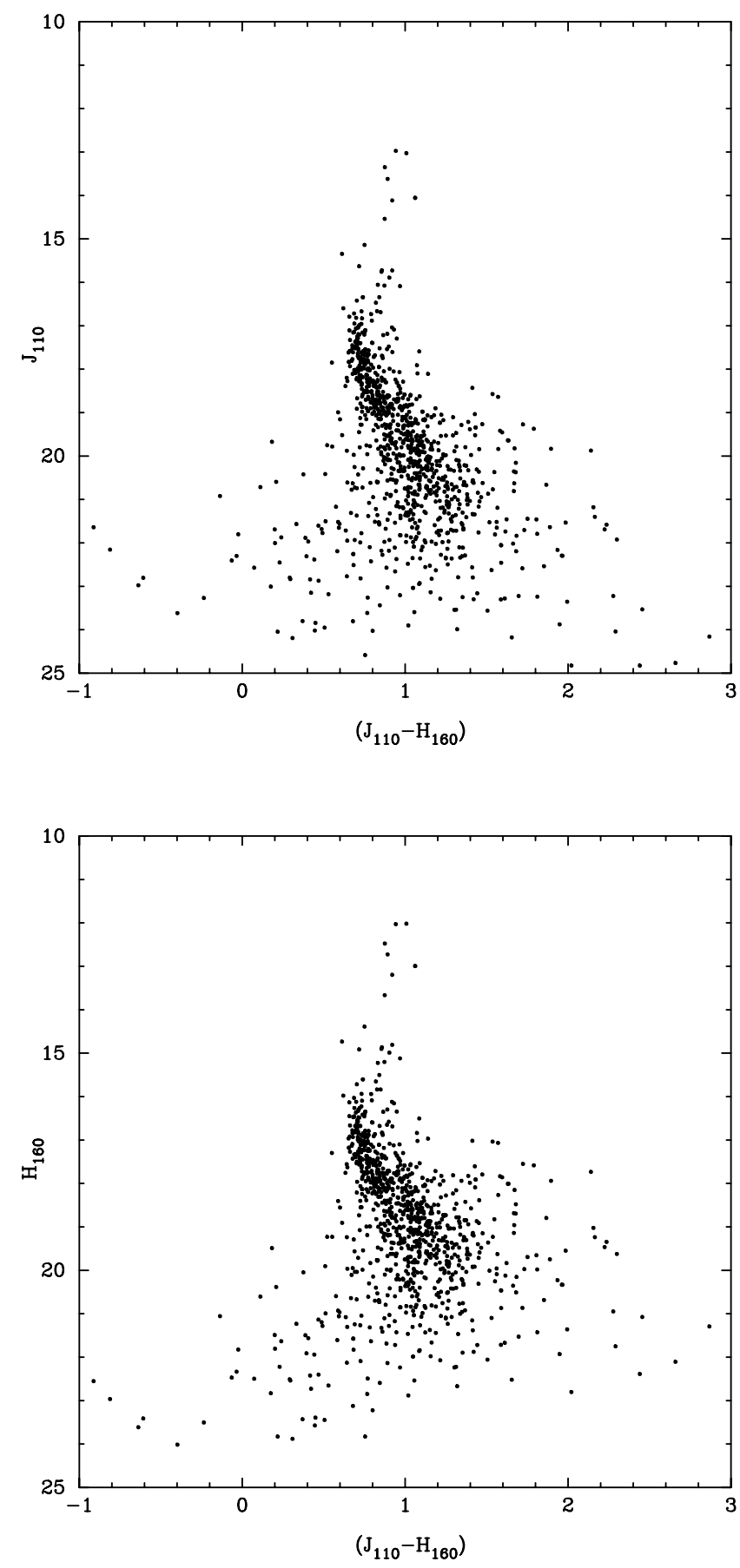

FIG. 19.-NICMOS NIC2 $J$ and $H$ color-magnitude diagrams for NGC 6553. The substantially reduced width of the main-sequence turnoff, relative to the optical color-magnitude data, show that reddening is a major contributor to the appearance of the photometry for this cluster.

F110W and F160W images. The width of the main sequence near the turn-off in these diagrams is substantially less than in the optical diagrams shown above, confirming that nonuniform extinction is indeed a factor contributing to the appearance of the color-magnitude diagrams for this cluster. This is most clearly illustrated by comparison of Figure 19 with Figure 20, showing the optical-infrared color-magnitude diagram. The substantially increased scatter in the width of the main sequence is apparent. These two-color relations remain a valuable resource for compari-
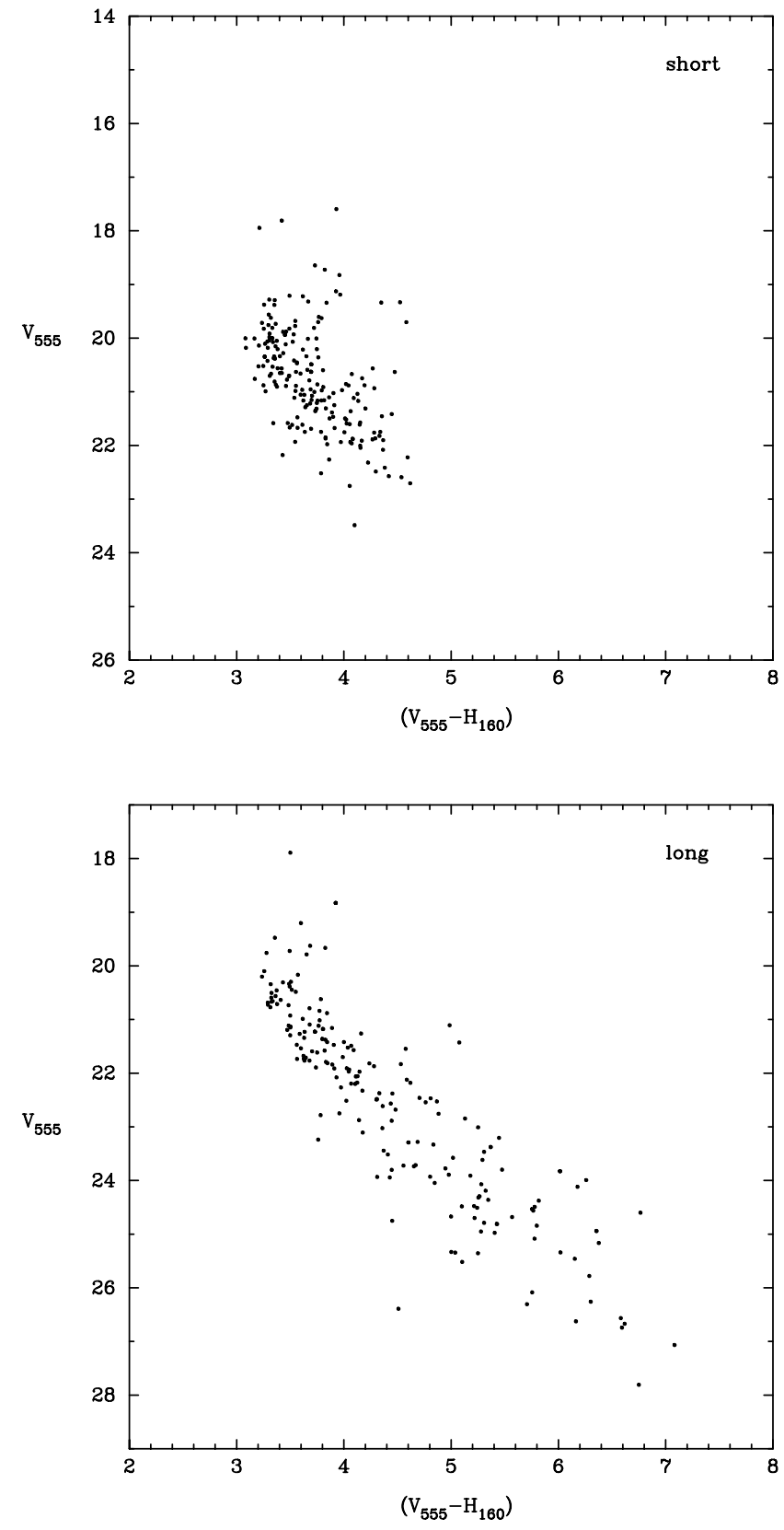

FIG. 20.-Optical and NICMOS color-magnitude relations for NGC 6553. The significant increase in width of the main sequence in the colormagnitude relations involving optical colors, compared with that in purely infrared colors, is indicative of variable extinction across the cluster, even on the scale of the NIC2 field of view.

son with metal-rich near-IR isochrones, in spite of this complexity.

For completeness, we also show here Figure 21, the optical-infrared two-color diagram. The points in this figure clearly do not scatter along the expected narrow single-age, single-abundance, single-extinction locus. Rather, a substantial minority of stars is seen to be offset along the direction of the reddening vector, by an amount of some $30 \%$ of the total extinction. Note that this variation is seen inside the very small NIC2 field of view. This confirms earlier suspicions that the color-magnitude diagram, luminosity function, etc., of NGC 6553 is significantly affected by patchy extinction. In agreement with our analysis of the 

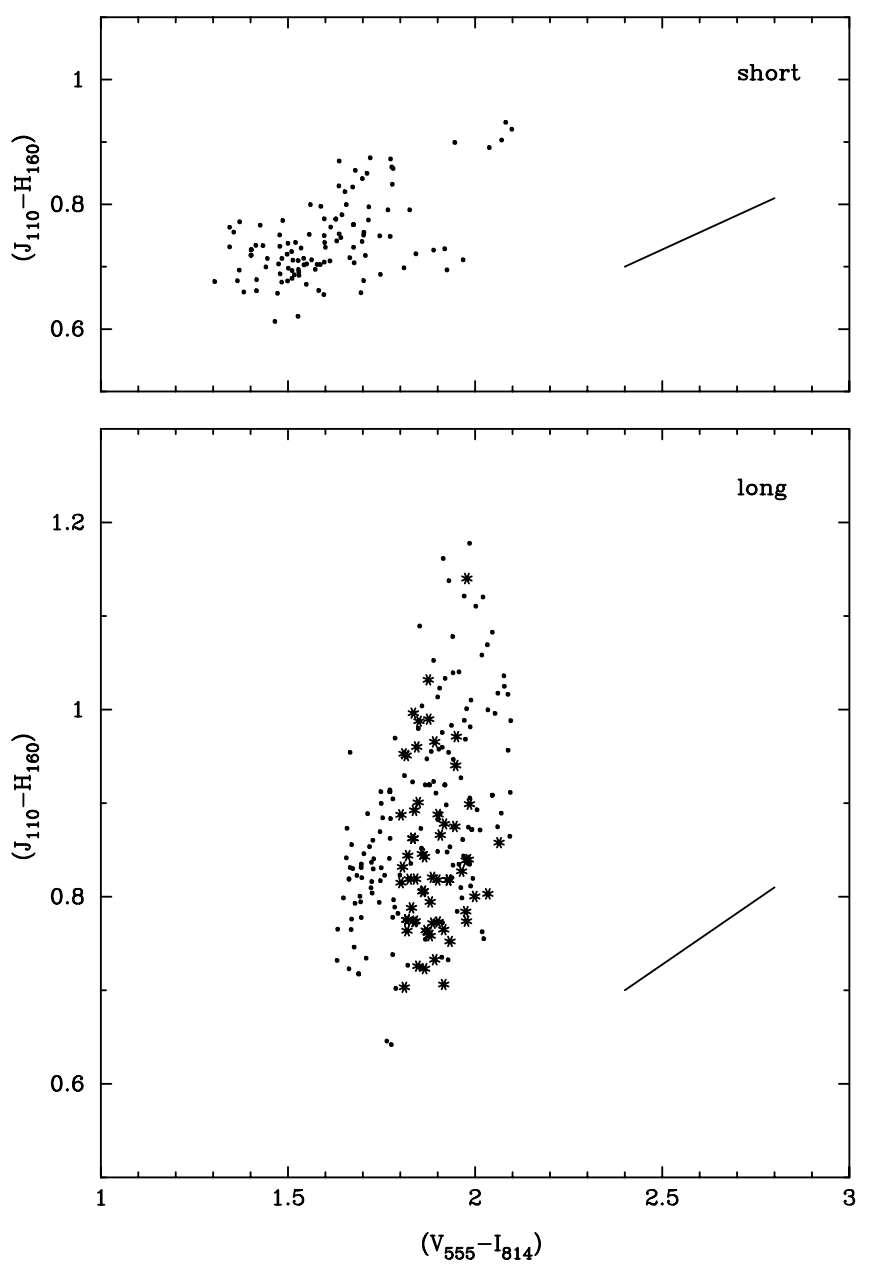

FIG. 21.-Optical-infrared two-color diagram for NGC 6553. The slope of the reddening vector is also shown. The top panel shows stars from the short exposure WFPC2 data, with $V<21, V-I<2.2$, essentially the main-sequence turnoff and lower RGB. The bottom panel shows data from the long exposure WFPC2 data, with $21.0<V<23.0, V-I<2.2$, essentially the main sequence just below the turnoff. Stars from the red flare, with $21.0<V<22.0, V-I>1.8$, are indicated by open star symbols. These stars are systematically bluer in $J-H$ than cluster stars of the same optical color, consistent with their being a lower metallicity background bulge population. Note also the significant width of the color distribution. This illustrates that part of NGC 6553 is affected by variable extinction of amplitude some $30 \%$ of the total line-of-sight extinction toward the cluster. It is this variable extinction which complicates analysis of the photometry for this cluster.

slope of the horizontal branch, however, not all stars and not all features of the color-magnitude diagram can be explained in this way.

\section{STIS-WFPC2 CALIBRATIONS}

Our primary goal in observing NGC 6553 as part of this project was to provide a calibration to convert STIS magnitudes to the much better studied WFPC2 system and hence to convert STIS apparent magnitudes for stars in the LMC

TABLE 6

PhOTOMETRIC CONVERSION COEFFICIENTS

\begin{tabular}{lccc}
\hline \hline Passband & $V_{555}-I_{814}$ & $\left(V_{555}-I_{814}\right)^{2}$ & ZP \\
\hline$V_{555} \cdots \cdots$ & -0.5184 & -0.0502 & -23.473 \\
$I_{814} \cdots \cdots$ & +0.4742 & -0.0487 & -23.464 \\
\hline
\end{tabular}

to stellar absolute luminosities in a standard system. With this in mind, NGC 6553 was chosen specifically because its metallicity is similar to those of young- and intermediateage LMC clusters.

\subsection{The Relationship between STIS $I_{L P}$ and WFPC2 Magnitudes}

Our STIS image of NGC 6553 intentionally overlaps the WFPC2 PC field, so that we can compare directly the magnitudes of the same set of stars derived from the two instruments. Figure 22 shows a comparison of STIS-LP with the long-exposure F555W and F814W magnitudes from 1646 and 1623 stars common to both samples respectively and the resulting color dependence of the STIS versus WFPC2 filter systems.

The relationship between the STIS $I_{\mathrm{LP}}$ and the WFPC2 $V_{555}$ and $I_{814}$ magnitude systems must be essentially linear, since all three are magnitudes with a zero-point offset and a color term to be determined. Since the STIS $I_{\mathrm{LP}}$ photometric system is very broad, we allow for this color term to be nonlinear, and we expect it to be a function not only of stellar absolute magnitude but also of stellar metallicity. It is for this reason that we have studied three calibration clusters with a range of metallicity. In this paper we consider NGC 6553 , with $[\mathrm{Fe} / \mathrm{H}] \approx-0.2$. In a companion paper (Houdashelt et al. 2001) we derive corresponding relationships for $\mathrm{M} 15([\mathrm{Fe} / \mathrm{H}] \approx-2.2)$ and for 47 Tuc $([\mathrm{Fe} / \mathrm{H}] \approx-0.7)$, and consider the metallicity-dependence of the resulting calibrations.

We expect to define relations of the following form:

$$
\begin{aligned}
& V_{555}=I_{\mathrm{LP}}+b_{1}\left(V_{555}-I_{814}\right)+c_{1}\left(V_{555}-I_{814}\right)^{2}+\mathrm{ZP}_{V} \\
& I_{814}=I_{\mathrm{LP}}+b_{2}\left(V_{555}-I_{814}\right)+c_{2}\left(V_{555}-I_{814}\right)^{2}+\mathrm{ZP}_{I}
\end{aligned}
$$

The coefficients in these fits were determined using an iterative $2 \sigma$ clipping algorithm and are presented in Table 6 , while the data and the fits to the data are shown in Figure 22.

\section{CONCLUSIONS}

The metal-rich globular cluster NGC 6553 has long been known to have a complex color-magnitude diagram. We have obtained HST optical (WFPC2 and STIS) and nearinfrared (NICMOS) observations of NGC 6553 to calibrate photometric conversions between the various HST passbands. In addition to that calibration, which we provide, we utilize the data to study the cluster. The optical colormagnitude diagram shows a curving $\mathrm{RGB}$, indicative of high metallicity, a tilted horizontal branch, a very broad main sequence, and a red flare of stars to the red of the main sequence fainter than the turnoff. We use our combined data to support earlier suggestions that variable extinction explains some, but not all, of these peculiarities. The horizontal branch is indeed tilted, at least in part, by an astrophysical cause. The width of the main sequence is caused in large part by patchy extinction. The red flare is, however, most consistent with being the background bulge field stars seen through the cluster. In this case, NGC 6553 is more metal-rich than is the mean field bulge star.

All conclusions relevant to this cluster, including ours, are seriously compromised by the large and patchy extinc- 

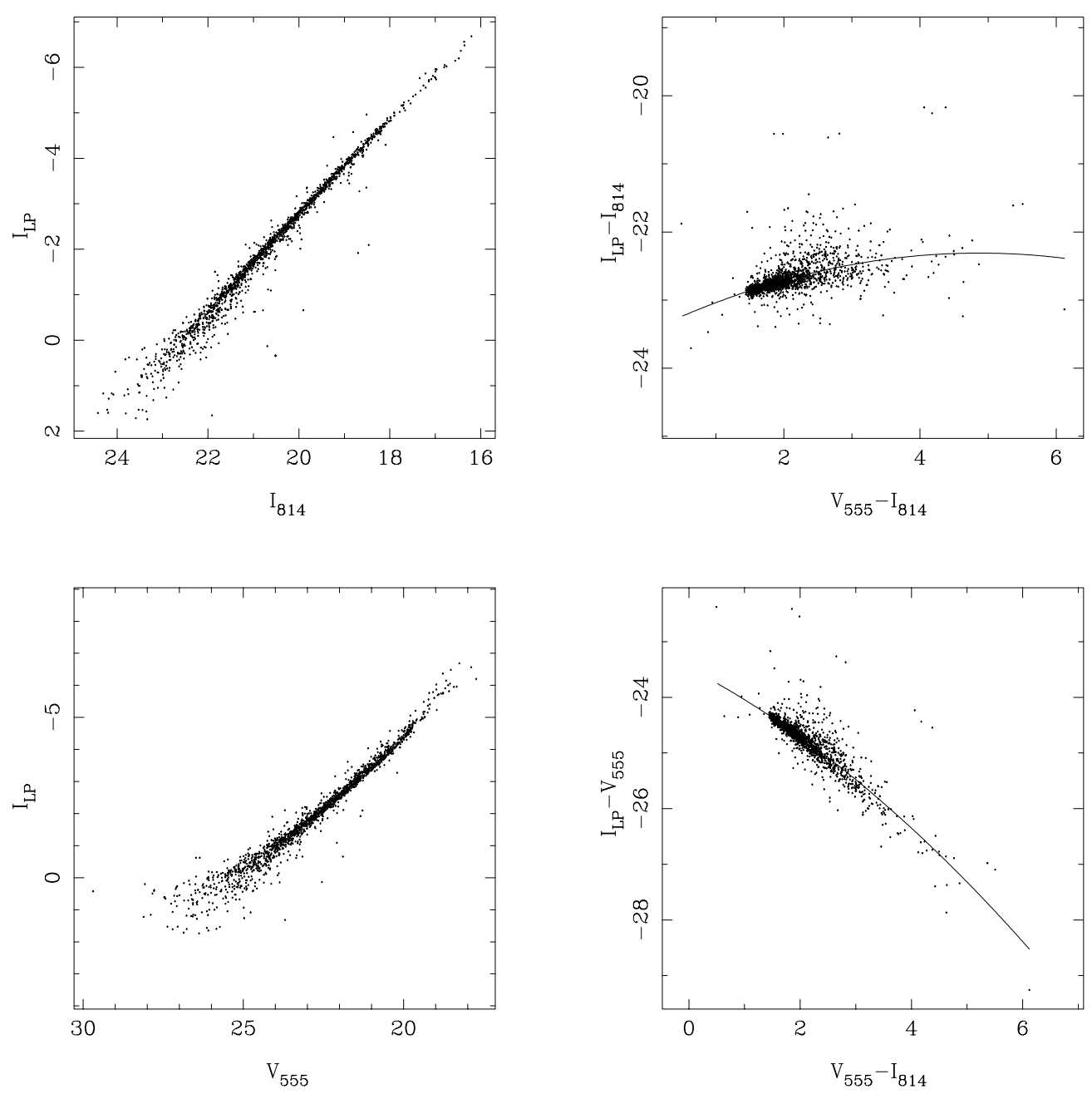

FIG. 22.-Comparison of STIS and WFPC2 magnitudes. The top left panel compares STIS $I_{\mathrm{LP}}$ and WFPC2 $I_{814}$ magnitudes (stars in common). The bottom left panel compares STIS $I_{\mathrm{LP}}$ and WFPC $2 V_{555}$ magnitudes (stars in common). In each case there are $\sim 1600$ stars in common. The panels on the right show the residual color dependence from the STIS vs. WFPC2 calibrations. The solid lines are the fits described in the text.

tion, allowing the possibility of significant residual systematic errors and making meaningless any formal error bars on derived quantities. NGC 6553 is affected by variable extinction of amplitude some $30 \%$ of the total line of sight extinction toward the cluster. It is this variable extinction that complicates analysis of the photometry for this cluster. Nonetheless, our best efforts to reproduce the photometric data, while remaining imperfect, suggest an age of $13 \mathrm{Gyr}$, a solar abundance and a distance modulus 0.4 mag smaller than that of previous studies. The extinction varies markedly on small scales, invalidating use of a single extinction in photometric studies supporting spectroscopic abundance determinations. Our isochrone study supports a near-solar abundance. This high metallicity is consistent with the general shape of the color-magnitude diagram.

\section{REFERENCES}

Aarseth, S. J. 1999, PASP, 111, 1333

Baggett, S., Casertano, S., Gonzaga, S., \& Ritchie, C. 1997, Instrument Science Report WFPC2 97-10 (Baltimore: STScI)

Barbuy, B., Bica, E., \& Ortolani, S. 1998, A\&A, 333, 117

Barbuy, B., Renzini, A., Ortolani, S., Bica, E., \& Guarnieri, M. D. 1999, A\&A, 341, 539

Beaulieu, S. F., Elson, R., Gilmore, G., Johnson, R. A., Tanvir, N., \& Santiago, B. 1999a, in IAU Symp. 190, New Views of the Magellanic Clouds, ed. Y.-H. Chu et al. (San Francisco: ASP), 460

Beaulieu, S. F., Gilmore, G., Johnson, R. A., Smartt, S. J., Tanvir, N., \& Santiago, B. 2000, in ASP Conf. Ser. 211, Massive Stellar Clusters (San Francisco: ASP), 128

Bertelli, G., Bressan, A., Chiosi, C., Fagotto, F., \& Nasi, E. 1994, A\&AS, 106, 275

Biretta, J., et al. 1996, WFPC2 Instrument Handbook (version 4.0; Baltimore: STScI)

Burleigh, M., Saffer, R., Gilmore, G., \& Napiwotzki, R. 1999, MNRAS, 310, L1

Castro, R., Santiago, B. X., Gilmore, G. F., Beaulieu, S. F., \& Johnson, R. A. 2001, MNRAS, in press

Carretta, E., Cohen, J. G., Gratton, R. G., \& Behr, B. B. 2001, AJ, submitted (astro-ph/0102014)

Cohen, J. G., Gratton, R. G., Behr, B. B., \& Carretta, E. 1999, ApJ, 523, 739

de Marchi, G. 1999, AJ, 117, 303

Dickinson, M., et al. 1999, NICMOS Data Handbook (version 4.0; Baltimore: STScI)

Elson, R. A. W., Gilmore, G. F., Santiago, B. X., \& Casertano, S. 1995, AJ, 110,682

Elson, R. A. W., Sigurdsson, S., Davies, M., Hurley, J., \& Gilmore, G. 1998a, MNRAS, 300, 857

Elson R. A. W., Sigurdsson S., Hurley J., Davies, M., \& Gilmore G. 1998b, ApJ, 499, L53

Elson, R., Tanvir, N., Gilmore, G., Johnson, R. A., \& Beaulieu, S. F. 1999, in IAU Symp. 190, New Views of the Magellanic Clouds, ed. Y.-H. Chu et al. (San Francisco: ASP), 417

Feltzing, S., \& Gilmore, G. 2000, A\&A, 355, 949 
Feltzing, S., Gilmore, G., \& Wyse, R. F. G. 1999, ApJ, 516, L17

Ferraro, F., et al. 1997, A\&A, 324, 915

Gardner, J. P., et al. 2000, AJ, 119, 486

Gilliland, R. L., Goudfrooij, P., \& Kimble, R. A. 1999, PASP, 111, 1009

Grebel, E. K., \& Roberts, W. J. 1995, A\&AS, 109, 293

Guarnieri, M. D., Ortolani, S., Montegriffo, P., Renzini, A., Barbuy B., Bica, E., \& Moneti, A. 1998, A\&A, 331, 70

Guathakurta, P., Webster, Z., Yanny, B., Schneider, D., \& Bahcall, J. 1998, AJ, 116, 1757

Harris, W. E. 1996, AJ, 112, 1487

Holtzman, J. A., et al. 1995, PASP, 107, 156

Houdashelt, M., Wyse, R. F. G., \& Gilmore, G. 2001, PASP, 113, 49

Johnson, R. A., Beaulieu, S. F., Elson, R. A. W., Gilmore, G., Tanvir, N., \& Santiago, B. 2000, in ASP Conf. Ser. 198, Stellar Clusters and Associations: Convection, Rotation, and Dynamos, ed. R. Pallavicini, G. Micela, \& S. Sciortino (San Francisco: ASP), 199

Johnson, R. A., Beaulieu, S. F., Gilmore, G. F., Hurley, J., Santiago, B. X., Tanvir N. R., \& Elson, R. A. W. 2001, MNRÄS, submitted

Johnson, R. A., Elson, R. A. W., Gilmore, G., Beaulieu, S., \& Tanvir, N. R. 1998, in NICMOS and the VLT: A New Era of High-Resolution Near Infrared Imaging and Spectroscopy, ed. W. Freudling \& R. Hook (Garching: ESO), 140

Lanteri Cravet, F., Guarneri, M., Renzini, A., \& Ortolani, S. 1997, in Advances in Stellar Evolution, ed. R. Rood \& A. Renzini (Cambridge: Cambridge Univ. Press), 59
Mandushev, G., Fahlman, G., Richer, H., \& Thompson, I. 1997, AJ, 114, 1060

Ortolani, S., Barbuy, B., \& Bica, E. 1990, A\&A, 236, 362

Ortolani, S., Renzini, A., Gilmozzi, R., Marconi, G., Barbuy, B., Bica, E., \& Rich, R. M. 1995, Nature, 377, 701

Olszewski, E. W., Schommer, R. A., Suntzeff, N. B., \& Harris, H. C. 1991, AJ, 101, 515

Paresce, F., \& de Marchi, G. 2000, ApJ, 534, 870

Piotto, G., Cool, A., \& King, I. 1997, AJ, 113, 1345

Piotto, G., \& Zoccali, M. 1999, A\&A, 345, 485

Rey, S. C., Lee, Y. W., Byun, Y. I., \& Chun, M. S. 1998, AJ, 116, 1775

Sagar, R., Subramaniam, A., Richtler, T., \& Grebel, E. K. 1999, A\&AS, 135, 391

Sahu, K., et al. 1999, STIS Instrument Handbook (version 3.0; Baltimore: STScI)

Santiago, B., Elson, R., \& Gilmore, G. 1996, MNRAS, 281, 1363

Santiago, B., Beaulieu, S., Johnson, R., \& Gilmore, G., 2001, A\&A, 369, 74

Sigurdsson, S., Davies, M. B., \& Bolte, M. 1994, ApJ, 431, L115

von Hippel, T., Gilmore, G., Tanvir, N., Robinson, D., \& Jones, D. 1996, AJ, 112, 192

Whitmore, B., Heyer, I., \& Casertano, S. 1999, PASP, 111, 1559

Wyse, R. F. G., Gilmore, G., \& Franx, M. 1997, ARA\&A, 35, 637

Zoccali, M., Renzini, A., Ortolani, S., Bica, E., \& Barbuy, B. 2001, preprint (astro-ph 0101200) 